\title{
乌ु \\ Spin-polarized electric currents in diluted magnetic semiconductor heterostructures induced by terahertz and microwave radiation
}

\author{
P. Olbrich, ${ }^{1}$ C. Zoth, ${ }^{1}$ P. Lutz, ${ }^{1}$ C. Drexler, ${ }^{1}$ V. V. Bel'kov, ${ }^{2}$ Ya. V. Terent'ev, ${ }^{2}$ S. A. Tarasenko, ${ }^{2}$ A. N. Semenov, ${ }^{2}$ S. V. Ivanov, ${ }^{2}$ \\ D. R. Yakovlev, ${ }^{2,3}$ T. Wojtowicz, ${ }^{4}$ U. Wurstbauer, ${ }^{5}$ D. Schuh, ${ }^{1}$ and S. D. Ganichev ${ }^{1}$ \\ ${ }^{1}$ Terahertz Center, University of Regensburg, 93040 Regensburg, Germany \\ ${ }^{2}$ Ioffe Physical-Technical Institute, Russian Academy of Sciences, 194021 St. Petersburg, Russia \\ ${ }^{3}$ Experimental Physics 2, TU Dortmund University, 44221 Dortmund, Germany \\ ${ }^{4}$ Institute of Physics, Polish Academy of Sciences, 02668 Warsaw, Poland \\ ${ }^{5}$ Columbia University, 435 W 116th St New York, New York 10027-7201, USA
}

(Received 27 April 2012; published 16 August 2012)

\begin{abstract}
We report on the study of spin-polarized electric currents in diluted magnetic semiconductor (DMS) quantum wells subjected to an in-plane external magnetic field and illuminated by microwave or terahertz radiation. The effect is studied in (Cd,Mn)Te/(Cd,Mg)Te quantum-wells (QWs) and (In,Ga)As/InAlAs:Mn QWs belonging to the well-known II-VI and III-V DMS material systems, as well as in heterovalent AlSb/InAs/(Zn,Mn)Te QWs, which represent a promising combination of II-VI and III-V semiconductors. Experimental data and developed theory demonstrate that the photocurrent originates from a spin-dependent scattering of free carriers by static defects or phonons in the Drude absorption of radiation and subsequent relaxation of carriers. We show that in DMS structures, the efficiency of the current generation is drastically enhanced compared to nonmagnetic semiconductors. The enhancement is caused by the exchange interaction of carrier spins with localized spins of magnetic ions resulting, on the one hand, in the giant Zeeman spin splitting, and, on the other hand, in the spin-dependent carrier scattering by localized $\mathrm{Mn}^{2+}$ ions polarized by an external magnetic field.
\end{abstract}

DOI: 10.1103/PhysRevB.86.085310

PACS number(s): 73.21.Fg, 72.25.Fe, 78.67.De, 73.63.Hs

\section{INTRODUCTION}

Transport of spin-polarized carriers in low-dimensional semiconductor structures is in the focus of intensive research aiming at spintronics. ${ }^{1-7}$ In particular, spin transport phenomena in diluted magnetic semiconductors (DMS) are currently discussed as a key issue for the development of semiconductorbased spintronic devices (see, e.g., Refs. 6 and 8-12). DMS materials represent semiconductors where paramagnetic ions, usually $\mathrm{Mn}$, are introduced in the host III-V or II-VI materials. ${ }^{13}$ The magnetic properties of the DMS structures can be widely tuned from paramagnetic to ferromagnetic behavior by varying concentration of magnetic ions, their location in the heterostructure, and by the structure fabrication. Strong "s $p-d$ " exchange interaction, which couples free carrier spins with the localized spins of magnetic ions, greatly enhances magnetooptical and magnetotransport effects in DMS structures. An important issue in the field of spin-dependent phenomena is the generation of spin currents or spin-polarized electric currents, e.g., due to electric spin injection, anomalous Hall effect, spin Hall effect, and spin-polarized tunneling. A further way to generate spin-polarized currents provides a spin-dependent scattering of free carriers excited by infrared or terahertz $(\mathrm{THz})$ radiation. This effect was observed in various low-dimensional nonmagnetic semiconductor structures ${ }^{9,14-16}$ and has been shown to be strongly enhanced in DMS structures. ${ }^{17}$ One of the advantages of these structures is that a nearly fully spin-polarized electric current may be generated due to the strong "sp-d" exchange interaction.

In this paper, we give a detailed theoretical description of spin-current mechanisms in DMS heterostructures. Experimental results are presented for DMS structures based on II-VI and III-V semiconductors as well as for hybrid II-VI/III-V heterostructures. We show that the exchange interaction in
DMS structures yields two roots to generate spin-polarized currents. One of them is related to the giant Zeeman splitting and the other one to the spin-dependent carrier scattering by localized magnetic ions polarized by an external magnetic field. The experimental results and the developed theory are in a good agreement, clearly showing that the current is spin polarized. The conclusion is strongly supported by the observed behavior of the photocurrent upon variation of temperature and magnetic field strength, being typical for the exchange interaction in DMS materials.

The paper is organized as follows. In Sec. II, we present a microscopic theory of optically induced spin-polarized currents in DMS structures. We discuss corresponding models and the current behavior upon the variation of parameters of optical excitation (photon energy and polarization), sample characteristics, and temperature. Section III describes the experimental technique and geometry of measurements as well as radiation sources used. In Sec. IV, we describe the design and parameters of the samples, present experimental data, and compare the results with theory. We start with the well-known DMS material quantum-well (QW) systems based on $n-(\mathrm{Cd}, \mathrm{Mn}) \mathrm{Te} /(\mathrm{Cd}, \mathrm{Mg}) \mathrm{Te}$ (Sec. IV A) and $p$-(In,Ga)As/InAlAs:Mn (Sec. IV B) and then introduce the results obtained on recently designed heterovalent hybrid $\mathrm{AlSb} / \mathrm{InAs} /(\mathrm{Zn}, \mathrm{Mn}) \mathrm{Te}$ structures with a two-dimensional electron gas. The paper is summarized in Sec. V.

\section{MICROSCOPIC MODEL}

The origin of spin-polarized current generation is spindependent scattering of free carriers by static defects or phonons at the Drude absorption of radiation and subsequent relaxation of carriers. ${ }^{9}$ This is due to spin-orbit interaction 
in gyrotropic media, such as InAs-, GaAs-, and CdTe-based two-dimensional structures, which give rise to linear in the wave-vector terms in the matrix element of scattering. The total matrix element of scattering can be thus presented by ${ }^{18}$

$$
V_{\boldsymbol{k}^{\prime} \boldsymbol{k}}=V_{0}+\sum_{\alpha \beta} V_{\alpha \beta} \sigma_{\alpha}\left(k_{\beta}+k_{\beta}^{\prime}\right),
$$

where the first term on the right-hand side describes the conventional spin-independent scattering, $\sigma_{\alpha}$ are the Pauli matrices, $\boldsymbol{k}$ and $\boldsymbol{k}^{\prime}$ are the initial and scattered wave vectors, and $\alpha$ and $\beta$ are the Cartesian coordinates. The spin-dependent scattering of electrons by static defects and phonons described by the last term in Eq. (1) is analyzed in Refs. 18-20. The linear in the wave-vector contributions stem from bulk and structure inversion asymmetry of QWs. The spin and electron momentum-dependent scattering results in an asymmetry of electron distribution in $\boldsymbol{k}$ space in each spin subband if the electron gas is driven out of equilibrium. The corresponding processes for the Drude absorption, which is accompanied by scattering, and energy relaxation are illustrated in Figs. 1(b) and 1(c), respectively. Thus, the spin-dependent scattering leads to the emergence of oppositely directed electron fluxes $\boldsymbol{i}_{ \pm 1 / 2}$ in the spin subbands. For zero magnetic field, the fluxes

(a)

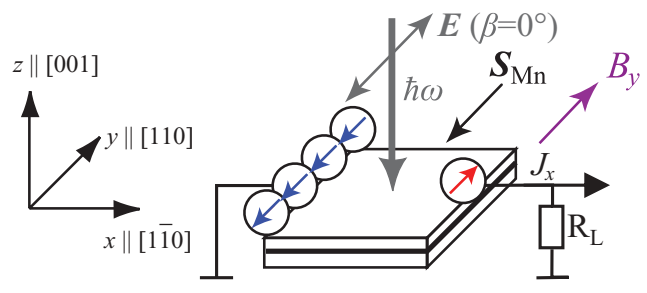

(b)

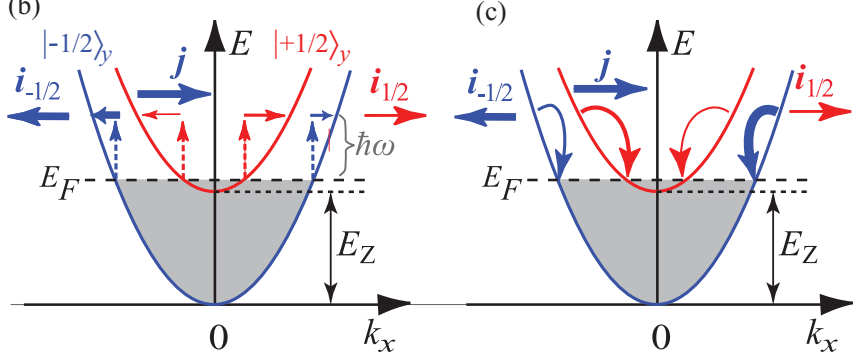

FIG. 1. (Color) Model of spin-polarized electric currents induced by terahertz/microwave radiation in DMS QW structures subjected to an in-plane magnetic field. (a) Illustration of the transverse electric current induced by the linearly polarized radiation at normal incidence and caused by the Zeeman splitting. The figure also sketches the typical experimental geometry where the electric current is measured by the voltage drop over the load resistance $R_{L}$. Arrows show directions of radiation electric field vector $\boldsymbol{E}$, magnetic field $B_{y}$, and average spin of $\mathrm{Mn}^{2+}$ ions $\boldsymbol{S}_{\mathrm{Mn}}$. Circles with oppositely directed arrows show electrons with opposite spins. (b) and (c) show the excitation and relaxation mechanisms of the current generation, respectively. Due to spin-dependent scattering, the transitions to the states with positive and negative $k_{x}$ in the spin subbands occur at different rates, which leads to the oppositely directed electron fluxes $\boldsymbol{i}_{+1 / 2}$ and $\boldsymbol{i}_{-1 / 2}$. This is illustrated for (b) scattering-assisted Drude absorption and (c) energy relaxation processes. The Zeeman splitting of the spin subbands results in their nonequal population. It disturbs the balance between the fluxes $\boldsymbol{i}_{+1 / 2}$ and $\boldsymbol{i}_{-1 / 2}$, giving rise to net spin-polarized electric current. are of equal magnitude forming a pure spin current defined as

$$
\boldsymbol{J}_{s}=(1 / 2)\left(\boldsymbol{i}_{+1 / 2}-\boldsymbol{i}_{-1 / 2}\right) .
$$

At nonzero magnetic field $\boldsymbol{B}$, the fluxes of electrons with the spin projections $\pm 1 / 2$ along $\boldsymbol{B}$ become unbalanced, giving rise to a net electric current $\boldsymbol{j}=e\left(\boldsymbol{i}_{+1 / 2}+\boldsymbol{i}_{-1 / 2}\right)$, where $e$ is the electron charge [see Figs. 1(a)-1(c)]. The microscopic calculation of the fluxes $\boldsymbol{i}_{ \pm 1 / 2}$ based on the Boltzmann approach is given in the Appendix.

A straightforward mechanism causing the electric current is the unequal population of the spin subbands due to the Zeeman effect. The mechanism is sketched in Figs. 1(b) and 1(c) for the scattering-assisted optical excitation (excitation mechanism) and relaxation (relaxation mechanism), respectively. In the case of photoexcitation [Fig. 1(b)], the transition rate in each spin subband depends on the subband population $n_{ \pm 1 / 2}$. Consequently, the electron fluxes in the spin subbands $\boldsymbol{i}_{ \pm 1 / 2} \propto$ $n_{ \pm 1 / 2}$ become unequal, resulting in the electric current

$$
\boldsymbol{j}_{Z}=4 \operatorname{es}(B) \boldsymbol{J}_{s},
$$

where $s(B)$ is the average electron spin projection along $\boldsymbol{B}$. For a low degree of spin polarization, it is given by

$$
s(B)=\frac{1}{2} \frac{n_{+1 / 2}-n_{-1 / 2}}{n_{+1 / 2}+n_{-1 / 2}}=-\frac{E_{\mathrm{Z}}}{4 \bar{E}} .
$$

Here, $E_{\mathrm{Z}}$ is the Zeeman splitting energy and $\bar{E}$ is the characteristic electron energy, equal to the Fermi energy $E_{\mathrm{F}}$ for degenerate two-dimensional electron gas (2DEG) and $k_{\mathrm{B}} T$ for nondegenerate gas at the temperature $T$, respectively. For linearly polarized radiation, photoexcited carriers are preferably aligned along the radiation electric field. Therefore, for a fixed magnetic field direction, e.g., $\boldsymbol{B} \| y$, the polarization plane rotation, described by the azimuth angle $\beta$, results in oscillations of the $x$ and $y$ current components as a function of $\beta$. Similarly, the Zeeman splitting gives rise to an electric current in the case of energy relaxation of hot electrons. Of course, the latter mechanism, which is based on electron gas heating, is independent of the radiation polarization. Microscopic and symmetry analyses show that it results in a current along the $x$ direction for $\boldsymbol{B} \| y$. For (001)-oriented QWs, the polarization dependencies of the total transverse $j_{x}$ and longitudinal $j_{y}$ photocurrents are given by (see Appendix)

$$
j_{x}=j_{1}+j_{2} \cos 2 \beta, \quad j_{y}=j_{3} \sin 2 \beta,
$$

where $j_{1}, j_{2}$, and $j_{3}$ are polarization-independent parameters, describing the relaxation mechanism $\left(j_{1}\right)$ and excitation mechanism $\left(j_{2}\right.$ and $\left.j_{3}\right), \beta$ is the azimuth angle $(\beta=0$ for $\boldsymbol{E} \| \boldsymbol{B})$, and $x \|[1 \overline{1} 0]$ and $y \|[110]$ are the Cartesian coordinates. Note that while the direction of the polarization-independent current is determined by the magnetic field direction, QW crystallographic orientation and design, the directions of the polarization-dependent components can be varied just by rotation of the polarization plane.

In diluted magnetic semiconductors, the considered mechanism of the photocurrent generation is drastically enhanced due to the giant Zeeman splitting. In DMS structures, the splitting is given by the sum of intrinsic and exchange contributions ${ }^{13}$

$$
E_{\mathrm{Z}}=g_{e(h)} \mu_{\mathrm{B}} B+\bar{x} S_{0} N_{0} \alpha_{e(h)} \mathrm{B}_{5 / 2}\left(\frac{5 \mu_{\mathrm{B}} g_{\mathrm{Mn}} B}{2 k_{\mathrm{B}}\left(T_{\mathrm{Mn}}+T_{0}\right)}\right),
$$



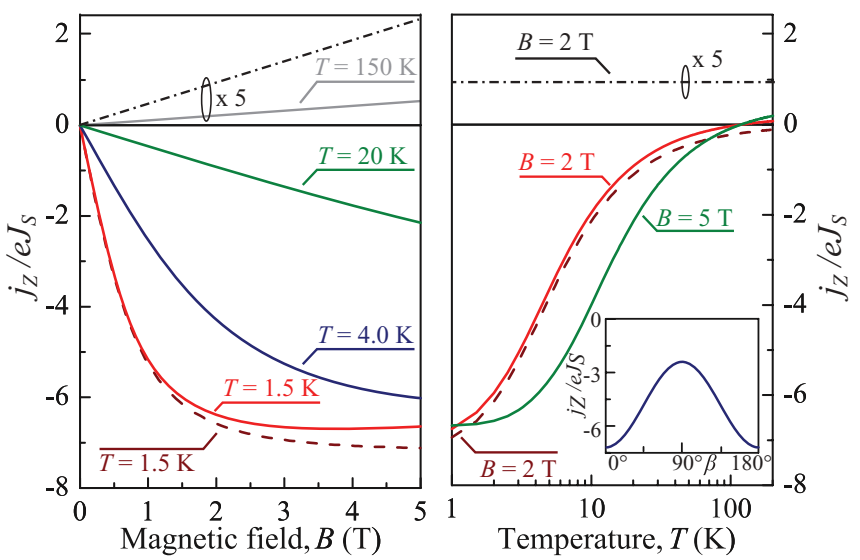

FIG. 2. (Color online) (a) Magnetic field dependence of the photocurrent contributions caused by the intrinsic and exchange Zeeman splitting and calculated after Eqs. (2) and (5) for different temperatures. (b) Temperature dependence of the photocurrent contributions calculated for different magnetic fields. Note that intrinsic contribution and the data at $T=150 \mathrm{~K}$ are multiplied by a factor of 5. Dotted-dashed line shows the current proportional to the intrinsic Zeeman splitting, which is here assumed to be temperature independent. Dashed line describes the current due to the exchange Zeeman splitting and respectively follows the Brillouin function $\mathrm{B}_{5 / 2}(\xi)$. Full lines correspond to the sum of the intrinsic and exchange contributions to the photocurrent calculated for different temperatures. Curves are obtained for literature values of parameters for $n-(\mathrm{Cd}, \mathrm{Mn}) \mathrm{Te} ; g_{e}=-1.64, \bar{x}=0.013, N_{0} \alpha_{e}=220 \mathrm{meV}, E_{\mathrm{F}}=$ $10 \mathrm{meV}$, and $T_{\mathrm{Mn}}=0$ (see Ref. 13). Inset shows an example of polarization dependence of the photocurrent given by Eq. (4).

where $g_{e(h)}$ is electron (hole) Landé factor in the absence of magnetic impurities, $\mu_{\mathrm{B}}$ is the Bohr magneton, $\bar{x}$ is the effective average concentration of $\mathrm{Mn}, N_{0} \alpha_{e(h)}$ is the exchange integral for conduction (valence) band carriers, $g_{\mathrm{Mn}}=2$ is $\mathrm{Mn} g$ factor, and $T_{\mathrm{Mn}}$ is the Mn-spin system temperature. Parameters $S_{0}$ and $T_{0}$ account for the Mn-Mn antiferromagnetic interaction, and $\mathrm{B}_{5 / 2}(\xi)$ is the modified Brillouin function.

Shown in Fig. 2 are magnetic field and temperature dependencies of the photocurrent $j_{e}$ calculated after Eqs. (2), (3), and (5) taking into account literature values of parameters for $n-(\mathrm{Cd}, \mathrm{Mn}) \mathrm{Te} \mathrm{QWs}$. In order to focus on the effect of magnetic impurities, we normalized the current $j_{e}$ by the pure spin current $J_{s}$, which depends on particular scattering mechanism and, therefore, may depend on temperature. At low temperatures, the current is dominated by the exchange interaction between free electrons and magnetic ions following the Brillouin function. As a result, the current first linearly grows with the magnetic field strength and then saturates. The increase in temperature leads to the decrease of the current magnitude and shifts the saturation to higher fields. Finally, at high temperatures, the exchange contribution to the Zeeman splitting becomes comparable or even smaller than the intrinsic one. In CdTe, where the sign of the intrinsic $g_{e}$ factor is opposite to the exchange one, this interplay results in a change of the photocurrent sign (see Fig. 2). ${ }^{21}$ In some other materials, e.g, $p$-type (In,Ga)As:Mn DMS structures, both contributions have the same sign ${ }^{3}$ and inversion does not occur.
Equation (3) yielding the linear relation between the average electron spin and the Zeeman splitting is valid for a low degree of electron gas polarization only. This regime is relevant for the majority of structures at moderate magnetic fields of several Tesla. However, in DMS structures, where a high degree of spin polarization can be achieved at moderate magnetic fields, the linear relation can be violated. This is another reason for the current saturation with rising the magnetic field. Indeed, in a fully spin-polarized electron gas, which can occur at low temperatures in DMS for magnetic fields even well below saturation of magnetization, the electron flux in one of the spin subbands vanishes. Therefore, the electric current becomes independent of the Zeeman splitting and is given by $\boldsymbol{j}=\mp 2 e \boldsymbol{J}_{s}$, where the sign is determined by the effective $g^{*}$-factor sign.

The described variation of the photocurrent with temperature and magnetic field is relevant for both the excitation and relaxation mechanisms sketched in Fig. 1. However, an effective way to distinguish between these microscopically different mechanisms is to study the polarization dependence of the photocurrent. Indeed, for a fixed magnetic field, the excitation-related photocurrent varies upon rotation the radiation polarization plane [see Eqs. (4)], while the relaxationrelated current does not. An example of the dependence of the current containing both contributions on the azimuth angle $\beta$ is shown in the inset in Fig. 2(b).

So far, we have considered mechanisms of the spinpolarized current formation based on the Zeeman splitting of electron spin subbands. However, there is an additional mechanism which leads to an imbalance between the fluxes $\boldsymbol{i}_{+1 / 2}$ and $\boldsymbol{i}_{-1 / 2}$ being specific for DMS structures. It is related to the spin-dependent electron scattering by polarized $\mathrm{Mn}^{2+}$ ions, which is described by the Hamiltonian of interaction between band electrons and magnetic ions ${ }^{13}$

$$
H_{e-\mathrm{Mn}}=\sum_{i}\left[u-\alpha\left(\hat{\boldsymbol{S}}_{i} \cdot \hat{\boldsymbol{S}}\right)\right] \delta\left(\boldsymbol{r}-\boldsymbol{R}_{i}\right) .
$$

Here, the index $i$ enumerates $\mathrm{Mn}$ ions, $\hat{\boldsymbol{S}}_{i}$ is the ion spin operator, $\hat{\boldsymbol{s}}=\boldsymbol{\sigma} / 2$ is the electron spin operator, $u \delta\left(\boldsymbol{r}-\boldsymbol{R}_{i}\right)$ is the scattering potential without exchange interaction, $\boldsymbol{r}$ the electron coordinate, and $\boldsymbol{R}_{i}$ the ion position. Note that the parameter $\alpha$ in Eq. (6) is also responsible for the giant Zeeman splitting in Eq. (5).

The scattering described by Eq. (6) provides a further mechanism for the generation of spin-polarized currents. As discussed above, the irradiation of gyrotropic QW structures causes two oppositely directed electron fluxes $\boldsymbol{i}_{ \pm 1 / 2}$. The external magnetic field polarizes the Mn spins leading to different scattering rates for band electrons with the spin projection $\pm 1 / 2$ along the ion polarization. ${ }^{22}$ Accordingly, the momentum relaxation times in the spin subbands $\tau_{p,+1 / 2}$ and $\tau_{p,-1 / 2}$ become unequal. Since the electron fluxes $\boldsymbol{i}_{ \pm 1 / 2}$ depend on the momentum relaxation times in the spin subbands, they do not compensate one another, giving rise to a net electric current $\boldsymbol{j}_{S c}$. This photocurrent can occur even for equally populated spin subbands and, therefore, is superimposed on the Zeeman splitting related contribution $\boldsymbol{j}_{Z}$. An estimation for $\boldsymbol{j}_{S c}$ can be made assuming that the momentum relaxation of electrons is determined by their interaction with $\mathrm{Mn}^{2+}$ ions. 
Taking into account the fact that the spin-independent part of the Mn potential, characterized by $u$, is usually much larger than the exchange term described by $\alpha$, we obtain

$$
\boldsymbol{j}_{S c}=2 e \tau_{p} \frac{\alpha}{u} \frac{\partial \boldsymbol{J}_{s}}{\partial \tau_{p}} S_{\mathrm{Mn}},
$$

where $\tau_{p}$ is the electron momentum relaxation time for the case of nonpolarized ions, $S_{\mathrm{Mn}}$ is the average Mn spin projection along $\boldsymbol{B}, S_{\mathrm{Mn}}=-S_{0} \mathrm{~B}_{5 / 2}(\xi)$, and $\boldsymbol{J}_{s}$ is formally considered as a function of $\tau_{p}$. Similarly to the current caused by the giant Zeeman splitting, the scattering-related current (7) is determined by the $\mathrm{Mn}$ ions polarization and, therefore, is characterized by nonlinear magnetic field dependence vanishing at high temperatures.

For a low degree of electron gas polarization, the photocurrent is given by the sum of two contributions

$$
\boldsymbol{j}=\boldsymbol{j}_{Z}+\boldsymbol{j}_{S c} .
$$

Due to the fact that both terms are caused by the exchange interaction, the resulting electric current will follow the Brillouin function no matter which contribution dominates. Depending on the structure material, the currents $\boldsymbol{j}_{Z}$ and $\boldsymbol{j}_{S c}$ may interfere in constructive or distractive ways. ${ }^{23}$ Possible ways to distinguish the relative contributions of $\boldsymbol{j}_{Z}$ and $\boldsymbol{j}_{S c}$ to the total spin-polarized electric current are to compare the temperature behavior of the current with that of the Zeeman splitting or to study the dependence of the current on the radiation frequency and structure mobility. Indeed, the first term in Eq. (8) is proportional to the total Zeeman spin splitting of electron states given by Eq. (5), while the second one is not. Thus, in the particular case of the intrinsic and exchange Landé factors being equal in magnitude but opposite in sign, the Zeeman splitting related contribution $j_{Z}$ vanishes, while the scattering contribution $j_{S c}$ remains. This situation in DMS structures can be obtained, e.g., by choosing the proper sample temperature.

Finally, we note that at high temperatures, where the exchange enhancement of the current is absent, additional orbital mechanisms may contribute to the magnetic-fieldinduced photocurrent. The orbital contribution comes from an asymmetry in the electron scattering due to the Lorentz force acting upon carriers. ${ }^{24,25}$ Its sign depends on the QW design and scattering mechanism. Therefore, the interplay of spin and orbital mechanisms may influence the current behavior, e.g., results in shifting the temperature inversion point or even its appearance/disappearance. The orbital contribution to the photocurrent may also show up at low temperatures and high magnetic fields. Being linear in the magnetic field, it may lead to a deviation of the field behavior of the measured current from the Brillouin function expected for the exchange mechanism.

\section{EXPERIMENTAL TECHNIQUE}

The experiments have been carried out on three different types of DMS low-dimensional structures with $\mathrm{Mn}^{2+}$ as the magnetic impurity. Here, spin-polarized photocurrents have been studied in the well-known II-VI and III-V DMS systems, represented by $n$-type $(\mathrm{Cd}, \mathrm{Mn}) \mathrm{Te} /(\mathrm{Cd}, \mathrm{Mg}) \mathrm{Te}$ QWs and $p$-type (In,Ga)As/InAlAs:Mn QWs, respectively, as well as in heterovalent hybrid II-VI/III-V n-type
$\mathrm{AlSb} / \mathrm{InAs} /(\mathrm{Zn}, \mathrm{Mn}) \mathrm{Te} \mathrm{QWs}$ with Mn layers inserted into the II-VI barriers. All structures have been grown by molecularbeam epitaxy on semi-insulating (001)-oriented GaAs substrates with buffer layers corresponding to each material group in order to relax strain.

A set of $5 \times 5 \mathrm{~mm}^{2}$ sized samples consisting of quantumwell structures with various densities and spatial position of Mn-doping layers have been prepared. To measure the photocurrent, two pairs of Ohmic contacts at the center of the sample edges oriented along the $x \|[1 \overline{1} 0]$ and $y \|$ [110] directions have been prepared [see inset in Fig. 4(b)]. The specific structures design and parameters are given in the beginning of the corresponding sections presenting the experimental results (see Secs. IV A-IV C). The samples were placed into an optical cryostat with $z$-cut crystal quartz windows and split-coil superconducting magnet. The magnetic field $B$ up to $7 \mathrm{~T}$ was applied in the QWs plane along the $y$ \| [110] axis. The sample temperature was varied from 1.8 up to $200 \mathrm{~K}$.

The experimental geometry is sketched in Fig. 1(a). The measurements of magnetic-field-induced photocurrents are carried out under excitation of the (001)-grown QW samples with linearly polarized terahertz and microwave radiation at normal incidence. The experimental arrangement is chosen to exclude any effects known to cause photocurrents at zero magnetic field. ${ }^{26}$ For optical excitation, we use four different types of radiation sources: low-power cw optically pumped $\mathrm{CH}_{3} \mathrm{OH} \mathrm{THz}$ laser, Gunn diodes, backwards wave oscillator, and high-power pulsed optically pumped $\mathrm{THz}$ laser. The sources provided monochromatic radiation in the frequency range between 0.1 and $\approx 2.5 \mathrm{THz}$ (corresponding photon energies, $\hbar \omega$ varied from 0.3 up to $10 \mathrm{meV}$ ). The radiation photon energies are smaller than the band gap as well as the size-quantized subband separation. Thus, the radiation induces indirect optical transitions in the lowest conduction subband (Drude-type free-carrier absorption).

Low-power excitation with $P \approx 2 \mathrm{~mW}$ at the sample spot is obtained by the $\mathrm{CH}_{3} \mathrm{OH} \mathrm{THz} \mathrm{laser-emitting} \mathrm{radiation} \mathrm{with}$ frequency $f=2.54 \mathrm{THz}$ (wavelength $\lambda=118 \mu \mathrm{m}$ ), ${ }^{27}$ backwards wave oscillator (Carcinotron) operating at $f=290 \mathrm{GHz}$ $(\lambda=1.03 \mathrm{~mm})$, and a Gunn diode with $f=95.5 \mathrm{GHz}$ $(\lambda=3.15 \mathrm{~mm})$. The incident power of the $\mathrm{cw}$ sources is modulated between 255 and $800 \mathrm{~Hz}$ by a PIN switch (Gunn diode) or an optical chopper. The photocurrent is measured across a $1-\mathrm{M} \Omega$ load resistor applying the standard lock-in technique. Pulsed high-power $\mathrm{THz}$ radiation with $f \approx 2.03 \mathrm{THz}(\lambda=$ $148 \mu \mathrm{m}$ ), a peak power $P \approx 40 \mathrm{~kW}$ at the sample spot, and a pulse duration of $\approx 200 \mathrm{~ns}$ is obtained by a $\mathrm{NH}_{3}$ laser optically pumped with a TEA $\mathrm{CO}_{2}$ laser. $^{28,29}$ In this setup, the signal is detected via a voltage drop over a $50-\Omega$ load resistor applying a fast amplifier and a storage oscilloscope. The radiation power has been controlled by either pyroelectric detectors or $\mathrm{THz}$ photon drag detector. The radiation is focused onto samples by one or two parabolic mirrors (for lasers and Carcinotron, respectively) or horn antenna (Gunn diode). Typical laser spot diameters varied, depending on the wavelength, from 1 to $3 \mathrm{~mm}$. The spatial beam distribution had an almost Gaussian profile, checked with a pyroelectric camera. The total power of the microwave radiation has been measured to be of the order of $10 \mathrm{~mW}$. However, the spatial distribution of the 
microwave radiation at the sample's position and its coupling to the sample could not have been determined with satisfactory accuracy. The main reasons for that were the strongly divergent microwave beam passing through the cryostat windows, and, in particular, the undefined geometry influencing efficiency of the radiation coupling to the sample, by, e.g., the bonding wires and metallization of contact pads. Thus, all microwave data are given in arbitrary units. In order to vary the angle $\beta$ between the light polarization plane and the magnetic field, the plane of polarization of the radiation incident on the sample was rotated by means of $\lambda / 2$ plates. Hereafter, the angle $\beta=$ $0^{\circ}$ is chosen in such a way that the incident light polarization is directed along the $y$ axis [see Fig. 1(a)].

\section{PHOTOCURRENT EXPERIMENTS}

In the following sections $(\mathrm{A}-\mathrm{C})$, we present the experimental results for three different groups of DMS low-dimensional structures. The sections are organized in a similar way: we start with the description of the structures design/parameters, then present a detailed study of the photocurrent behavior upon variation of the magnetic field strength, temperature, radiation intensity, and polarization, and, finally give a comparison of the results with the theory described in Sec. II.

\section{A. $n$-(Cd,Mn) Te/(Cd,Mg)Te quantum wells}

Low-dimensional structures based on wide-band-gap IIVI diluted magnetic semiconductors are the best understood DMS materials with the most studied electric and magnetic properties, ${ }^{8}$ and it is the DMS system in which the terahertz-radiation-induced spin-polarized electric current has been reported. ${ }^{17,30}$ The experiments presented below have been carried out on 10-nm-wide $n$-type $(\mathrm{Cd}, \mathrm{Mn}) \mathrm{Te}$ single QWs embedded in $(\mathrm{Cd}, \mathrm{Mg}) \mathrm{Te}$ barriers. The DMS QWs were grown by molecular-beam epitaxy on (001)-oriented GaAs substrates. ${ }^{22,31,32}$ Three groups of $n$ - $(\mathrm{Cd}, \mathrm{Mn}) \mathrm{Te} / \mathrm{Cd}_{0.76} \mathrm{Mg}_{0.24} \mathrm{Te}$ structures with different $\mathrm{Mn}$ contents (A0, A1, and A2) were fabricated. In each group, several samples from the same wafer were investigated. In the following, we discuss the data obtained on the nonmagnetic reference QWs (sample A0) and DMS QWs having different magnetic properties. In samples A1 and A2, several evenly spaced $\mathrm{Cd}_{1-x} \mathrm{Mn}_{x} \mathrm{Te}$ thin layers were inserted during the growth of the 10-nm-wide QW applying the digital alloy

(a) Sample A1 $\bar{x}=0.013$

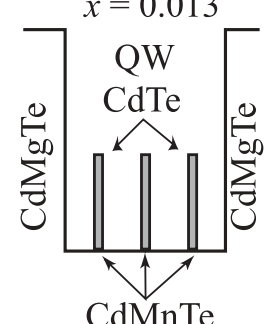

(b)

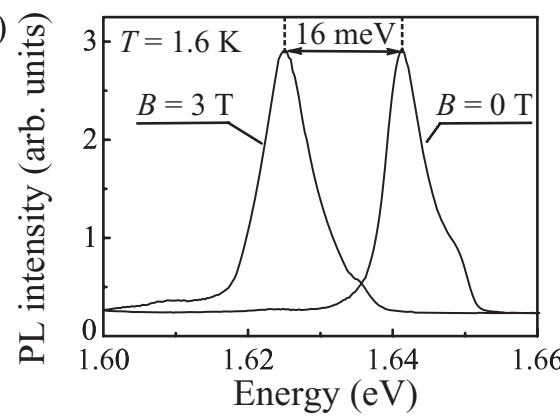

FIG. 3. Design and PL data for sample A1: (Cd,Mn)Te/ (Cd,Mg)Te DMS quantum-well structure. (a) Sketch of the structure. (b) Photoluminescence spectra at $B=0$ and $3 \mathrm{~T}$.
TABLE I. Parameters of A0-A2 samples. The effective average concentration of $\mathrm{Mn} \bar{x}$ is estimated from the giant Zeeman shift of the interband emission line. Mobility $\mu$ and electron sheet density $n_{e}$ data are obtained at $4.2 \mathrm{~K}$ in the dark.

\begin{tabular}{lccccc}
\hline \hline Sample & $x$ & $\bar{x}$ & $\mu\left(\mathrm{cm}^{2} / \mathrm{Vs}\right)$ & $n_{e}\left(\mathrm{~cm}^{-2}\right)$ & $E_{F}(\mathrm{meV})$ \\
\hline A0 & 0 & 0 & 59000 & $4.2 \times 10^{11}$ & 10.4 \\
A1 & 0.14 & 0.013 & 16000 & $6.2 \times 10^{11}$ & 15.4 \\
A2 & 0.20 & 0.015 & 9500 & $4.7 \times 10^{11}$ & 11.7 \\
\hline \hline
\end{tabular}

technique. ${ }^{33}$ In those samples, the spin splitting can be described using Eq. (5).

The sketch of sample A1 with three single monolayers of $\mathrm{Cd}_{0.86} \mathrm{Mn}_{0.14} \mathrm{Te}$ is shown in Fig. 3(a). Sample A2 has similar design but is fabricated with two insertions of three monolayers of $\mathrm{Cd}_{0.8} \mathrm{Mn}_{0.2} \mathrm{Te}$. In the II-VI semiconductor compound, the $\mathrm{Mn}$ atoms substitute the $\mathrm{Cd}$ atoms and provide a localized spin $S=5 / 2$. In order to obtain a two-dimensional electron gas, the structures have been modulation doped by iodine donors introduced into the top barrier at $15 \mathrm{~nm}$ distance from the QW. The electron density $n_{e}$ and mobility $\mu$ obtained by magnetotransport measurements, as well as the effective average concentration of $\mathrm{Mn} \bar{x}$ and the Fermi energy $E_{\mathrm{F}}$, estimated from the photoluminescence (PL) spectra, are summarized in Table I. PL spectra obtained from sample A1 at $B=0$ and $3 \mathrm{~T}$ is shown in Fig. 3(b). Here, the line for $B=$ $3 \mathrm{~T}$ is substantially red-shifted (about $16 \mathrm{meV}$ at $T=1.6 \mathrm{~K}$ ) relative to that for zero field. This shift corresponds to $32-\mathrm{meV}$ giant Zeeman splitting of band states from which $6.4 \mathrm{meV}$ fall into conduction band. ${ }^{13}$

We start by describing the results obtained with low-power $\mathrm{THz}$ and $\mathrm{mw}$ sources. The signal in unbiased samples is observed under normal incidence with linearly polarized radiation for both transverse and longitudinal geometries, where the current is measured in the direction perpendicular, $J_{x}$, and parallel, $J_{y}$, to the magnetic field $B_{y}$, respectively. ${ }^{34}$ Figure 4 shows magnetic field dependence of the transverse

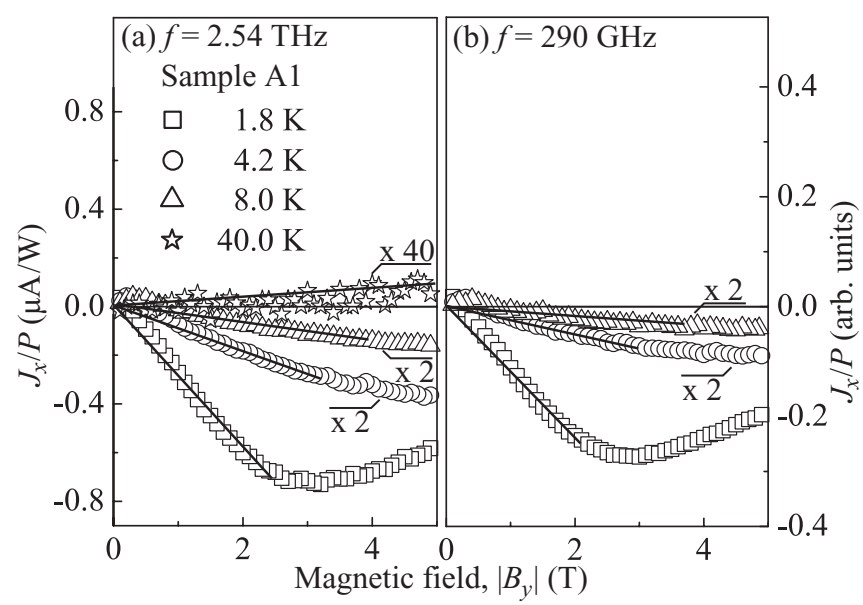

FIG. 4. Magnetic field dependence of the photocurrent measured in $(\mathrm{Cd}, \mathrm{Mn}) \mathrm{Te} /(\mathrm{Cd}, \mathrm{Mg}) \mathrm{Te}$ DMS QW sample A1 at various temperatures and applying (a) $\mathrm{THz}$ radiation, $f=2.54 \mathrm{THz}$ and (b) $\mathrm{mw}$ radiation, $f=290 \mathrm{GHz}$. Solid lines are linear fits for low $B$ as guides for the eye. 
photocurrent $J_{x}$. The detected photocurrent is an odd function of the magnetic field: It increases with raising magnetic field strength, vanishes for $B=0$, and its sign depends on the magnetic field direction. The signal linearly scales with the radiation power and does not show a hysteretic behavior as ensured by sweeping magnetic field from positive to negative fields and back (both not shown). For convenience, in the discussion below we evaluate the data after

$$
J_{x, y}\left(\left|B_{y}\right|\right)=\frac{J_{x, y}\left(B_{y}>0\right)-J_{x, y}\left(B_{y}<0\right)}{2},
$$

which yields solely the strength of the magnetic-field-induced photocurrent. Characteristic dependencies of the photocurrent upon variation of temperature, magnetic field strength, radiation wavelength, intensity, and polarization are the same for all samples within each group and are qualitatively the same for all DMS samples belonging to A1 and A2 groups. Thus, in the following we consistently present the data obtained on one of the A1 samples. Figures 4(a) and 4(b) show the magnetic field dependence of the transverse photocurrent $J_{x} / P$ measured in sample A1 under excitation with $\mathrm{cw}$ THz radiation ( $f=$ $2.54 \mathrm{THz})$ and $\mathrm{mw}$ radiation $(f=290 \mathrm{GHz})$, respectively. The experiments reveal that at high temperatures, or at low temperatures and moderate magnetic fields, the magnitude of $J_{x}$ is proportional to $B_{y}$ (see Ref. 35). At low temperatures and high magnetic fields, however, the photocurrent saturates with increasing $B_{y}$. Moreover, at $T=1.8 \mathrm{~K}$, a small reduction of signal with increasing magnetic field is observed for $B \gtrsim 3.5 \mathrm{~T}$.

Figure 5 shows the polarization dependence of the photocurrent measured in DMS sample A1 excited by $\mathrm{cw} \mathrm{THz}$ radiation. The data are obtained for $B_{y}= \pm 2 \mathrm{~T}$ at which the photocurrent does not show a saturation in the whole

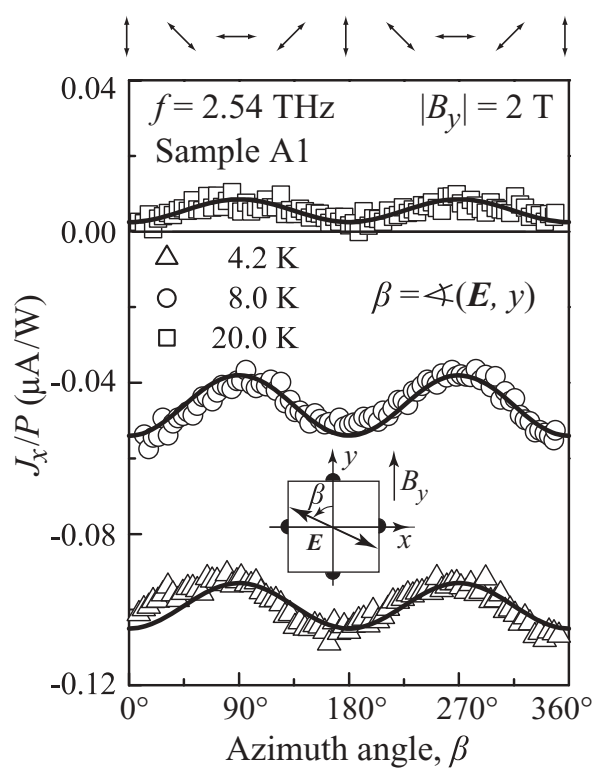

FIG. 5. Polarization dependencies of photocurrent measured in (Cd,Mn)Te/Cd,Mg)Te DMS QW sample A1 at fixed magnetic field $\left|B_{y}\right|=2 \mathrm{~T}$ and normal incidence of $\mathrm{THz}$ radiation $(f=2.54 \mathrm{THz})$ for $T=4.2,8$, and $20 \mathrm{~K}$. Fits are after Eqs. (4) with $j_{1}$ and $j_{2}$ as scaling parameters. The arrows on top show the orientation of the light's electric field. The inset defines the azimuth angle $\beta$.

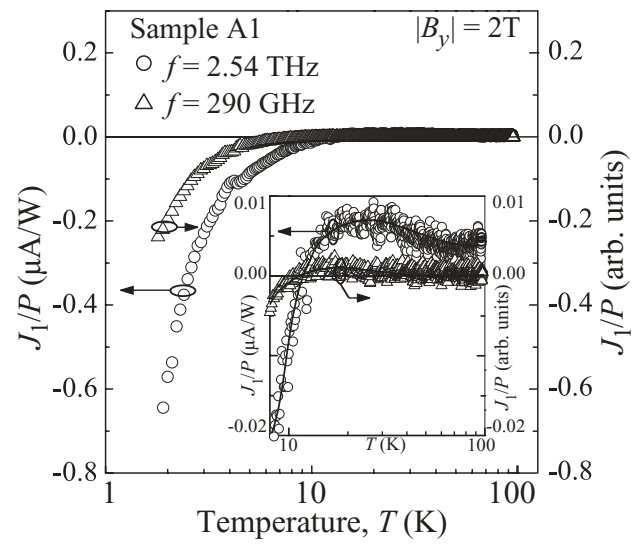

FIG. 6. DMS (Cd,Mn)Te/(Cd,Mg)Te sample A1: Temperature dependence of photocurrent (polarization-independent) at magnetic field $\left|B_{y}\right|=2 \mathrm{~T}$ and normal incidence of mw radiation $(f=$ $290 \mathrm{GHz})$ and $\mathrm{THz}$ radiation $(f=2.54 \mathrm{THz})$. The inset shows a zoom of $J_{x}(T)$ near the inversion point with solid lines as guides for the eye.

temperature range [see Fig. 4(a)]. Consequently, the signal behavior upon variation of the azimuth angle or temperature is not affected by the photocurrent saturation. The current $J_{x}$ is well described by the first equation of Eqs. (4) [see also inset in Fig. 2(b)] and consists of polarization-independent $J_{1}$ and polarization-dependent $J_{2} \cos (2 \beta)$ components. Following Eq. (4) the individual contributions to the transverse photocurrent, $J_{1}$ and $J_{2}$, can be deduced from the experiment by taking, respectively, a half-sum or a half-difference of the signals obtained at $\beta=0^{\circ}$ and $90^{\circ}$. In the longitudinal configuration, we detected only the polarization-dependent photocurrent $J_{y}=J_{3} \sin (2 \beta)$ well described by the second equation of Eqs. (4).

The most striking observation comes from the investigation of the temperature dependence of the polarization-independent photocurrent $J_{1}$. Figure 6 reveals that a cooling of the sample from $100 \mathrm{~K}$ down to $1.8 \mathrm{~K}$ results in, on the one hand, a change of the current direction, and, on the other hand, an increase of the photocurrent strength by about two orders of magnitude. Such a temperature dependence is observed for both the THz- and mw-radiation-induced photocurrents and the corresponding data differ by a scalar factor only (see Fig. 6). By contrast, in the reference nonmagnetic sample A0, the drastic enhancement of the signal magnitude and the inversion of the photocurrent direction with the temperature decrease have not been observed (not shown).

The peculiar temperature behavior observed in DMS QWs excited by low-power radiation dwindles under application of high-power pulsed $\mathrm{THz}$ radiation with $P \approx 40 \mathrm{~kW}$ (see Ref. 36). While at low-power excitation the photocurrent direction changes upon cooling and its magnitude strongly depends on the temperature (Figs. 4 and 6), the current induced by high-power pulsed $\mathrm{THz}$ radiation neither undergoes an inversion nor exhibits a significant dependence on $T$ in the range between 1.8 and $100 \mathrm{~K}$ (see Fig. 7). Furthermore, irradiation with high power leads to a strong decrease of the magnitude of signal normalized by the radiation power $J_{x} / P$ compared to the one for low-power data $(\approx 1 \mathrm{nA} / \mathrm{W}$ instead of $\approx 1 \mu \mathrm{A} / \mathrm{W})$. Moreover, the photocurrent saturation with 


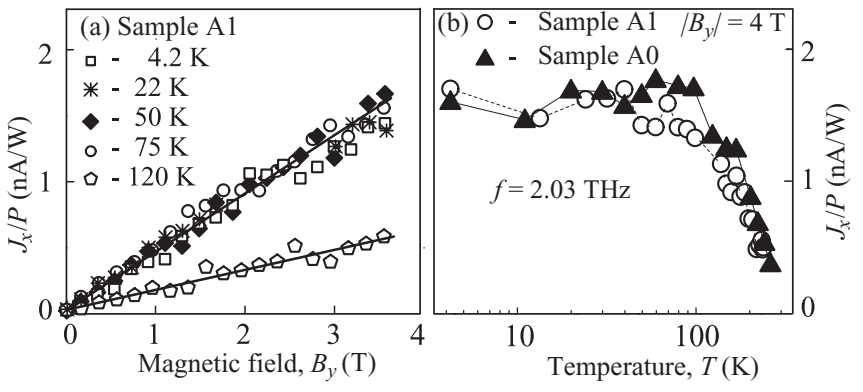

FIG. 7. (a) Magnetic field dependence of photocurrent at different temperatures for DMS $(\mathrm{Cd}, \mathrm{Mn}) \mathrm{Te} /(\mathrm{Cd}, \mathrm{Mg}) \mathrm{Te}$ sample A1 with linear in $B$ fit as guide for the eye and (b) temperature dependence at fixed magnetic field for reference CdTe sample A0 and DMS $(\mathrm{Cd}, \mathrm{Mn}) \mathrm{Te} /(\mathrm{Cd}, \mathrm{Mg}) \mathrm{Te}$ sample $\mathrm{A} 1$. The data are obtained at normal incidence of pulsed $\mathrm{THz}$ radiation $(f=2.03 \mathrm{THz})$ with powers up to $P \approx 40 \mathrm{~kW}$.

increasing magnetic field observed at low power disappears, and the signal excited by high-power laser linearly scales with magnetic field strength [Fig. 7(a)]. It is also remarkable that now DMS samples and nonmagnetic samples show the same temperature dependence: The photocurrent is almost independent of the sample temperature below about $100 \mathrm{~K}$ and decreases for $T>100 \mathrm{~K}$ [see Fig. 7(b)].

The experimental results described above are in a good agreement with the theory of radiation-induced spin-polarized electric currents in DMS quantum wells subjected to an in-plane external magnetic field (see Sec. II). Comparison of the photocurrent calculated after Eqs. (2) to (5) and shown in Fig. 2, with the corresponding data (see Figs. 4-6), shows qualitative similarity of the theoretical and experimental results. In particular, the drastic enhancement of the photocurrent magnitude and the change of its direction upon sample cooling, as well as the observed saturation of the signal with raising magnetic field strength, are clear consequences of the exchange interaction between the $s$-type conduction band electrons and the half-filled $d$ shell of the $\mathrm{Mn}^{2+}$ ions. The observed photocurrent sign inversion upon temperature variation is caused by the opposite signs of the intrinsic and exchange Zeeman spin splittings, well known for these materials. Due to the strong dependence of the Brillouin function $\mathrm{B}_{5 / 2}$ [see last term in Eq. (5)] on temperature sample heating results in the rapid reduction of the exchange part to the photocurrent and the dominance of the intrinsic one. The interplay of intrinsic and exchange $g$ factors contributes also to the deviation from the saturation behavior observed at low temperatures. Here, instead of the saturation expected for the Brillouin function, a slight decrease of the photocurrent at high magnetic fields is detected (see Fig. 4). Similar behavior is seen for the calculated photocurrent shown by the solid lines in Fig. 2(a), where both exchange and intrinsic contributions are taken into account (see Ref. 37).

While for low-power radiation the heating of the sample or the manganese system plays no essential role and the signal linearly scales with radiation intensity, a substantial increase of the radiation power qualitatively changes the photocurrent formation. Indeed, in the high-power experiments, neither an inversion nor a photocurrent enhancement by cooling down the sample have been observed (see Fig. 7). This indicates that at these conditions the polarization of the $\mathrm{Mn}^{2+}$ spins does not contribute to the generation of current. Figure 7 demonstrates that the photocurrent in DMS samples excited by high-power radiation is at all temperatures proportional to the magnetic field and varies with temperature in the same manner as the one measured in nonmagnetic reference sample A0. It can be well described with Eqs. (2), (3), and (5) assuming vanishing contribution of the exchange interaction. For low temperatures and degenerated electron gas, the characteristic electron energy $\bar{E}$ is equal to $E_{\mathrm{F}}$ and the photocurrent is nearly independent of $T$. In the case of a nondegenerated gas (higher temperatures), $\bar{E}$ is given by $k_{\mathrm{B}} T$ and leads to a $1 / T$ dependence of $J_{x}$. These two regimes are clearly pronounced in Fig. 7(b) and, in fact, are well known for spin-polarized photocurrents in nonmagnetic semiconductor structures. ${ }^{14,38}$

The observed photocurrent variation with the orientation of the radiation polarization plane is also in agreement with the theory developed in Sec. II. The polarization dependence of the transverse photocurrent shown in Fig. 5 is in agreement with Eq. (4) and the corresponding calculated curve shown in the inset in Fig. 2(b). It demonstrates that this current is a result of superposition of the polarization-independent current due to energy relaxation of hot electrons described by $j_{1}$ in Eqs. (4) (relaxation contribution) and the polarization-dependent one due to excitation given by the last term in the first equation in Eqs. (4). The longitudinal photocurrent is also observed and its polarization dependence is in agreement with the second equation in Eqs. (4).

The interplay of the giant exchange Zeeman splitting and the intrinsic one in the total spin splitting explains qualitatively the behavior of the photocurrent upon changing magnetic field strength, temperature, $\mathrm{Mn}$ doping, as well as radiation intensity and polarization. However, the observed increase of the current strength at low temperatures is substantially larger than the giant Zeeman shift measured in the same structures by the photoluminescence data. For example, in sample A1 at $B=3 \mathrm{~T}$, the spin splitting, derived from PL data, changes from $-0.25 \mathrm{meV}$ at high temperatures (intrinsic value given by $g_{e} \mu_{\mathrm{B}} B$ ) to $2.6 \mathrm{meV}$ at $4.2 \mathrm{~K}$ and, hence, its magnitude swells by about a factor 10 . By contrast, the magnitude of the photocurrent at $T=4.2 \mathrm{~K}$ increases by about factor of 100 compared to that measured for $T=40 \mathrm{~K}$ [see Fig. 4(a)]. This quantitative disagreement together with the strong temperature dependence of the signal provide an evidence for the dominating contribution of another DMS specific mechanism. This is the spin current due to the spin-dependent electron scattering by polarized $\mathrm{Mn}^{2+}$ ions which was elaborated at the end of Sec. II and is shown to amplify the current conversion vastly.

\section{B. Mn-doped $p$-(In,Ga)As/InAlAs quantum-well structures}

The second type of investigated samples III-V based DMS, with $\mathrm{Mn}$ as the magnetic impurity, is studied to a lesser extent than the principal II-VI DMS family, but are already well understood. ${ }^{8}$ Currently, this type of DMS structures is intensively studied because of their prospect for spin-polarized carrier injection $^{10-12,39}$ required for spintronics applications. In III-V semiconductors, such as InAs or GaAs, Mn atoms substitute the group-III elements (In, Al, or $\mathrm{Ga}$ ), providing 
(a) Sample B0

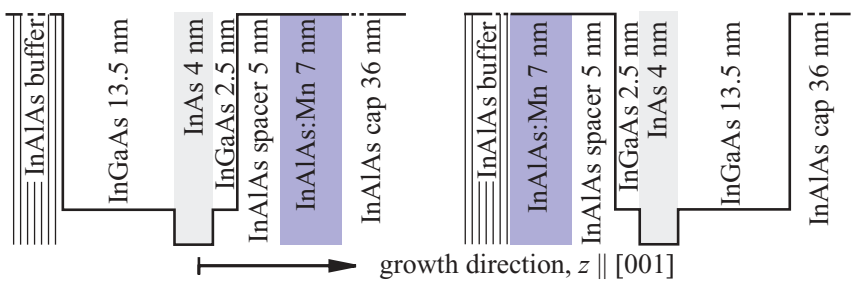

FIG. 8. (Color online) Sketch of the Mn-doped (In,Ga)As/ InAlAs samples with (a) normal-doped reference structure B0 and (b) inverted-doped DMS structure B1 for which segregation along growth direction results in a $\mathrm{Mn}$ ion penetration into the InAs QW.

both localized magnetic moments with spin $S=5 / 2$ and free holes ${ }^{40}$ in contrast to II-VI materials, where Mn is an isoelectric impurity.

Samples investigated in this work are compressively strained InAs quantum wells embedded in (In,Ga)As/ InAlAs:Mn host material with an In mole fraction of $75 \%$ (for details, see Ref. 41). High-mobility Mn modulation-doped single QW structures were grown by molecular-beam epitaxy on semi-insulating GaAs (001)-oriented substrates. The layer sequences of two fabricated Mn-doped samples are depicted schematically in Fig. 8. The active layer consists of a 20-nm $\mathrm{In}_{0.75} \mathrm{Ga}_{0.25}$ As channel with an additional strained 4-nm InAs $\mathrm{QW}$, a 5-nm thick $\mathrm{In}_{0.75} \mathrm{Al}_{0.25} \mathrm{As}$ spacer, a 7-nm thick Mndoped $\mathrm{In}_{0.75} \mathrm{Al}_{0.25}$ As layer, and a $36-\mathrm{nm} \mathrm{In}_{0.75} \mathrm{Al}_{0.25} \mathrm{As}$ cap layer. The samples differ in the position of the Mn-doped layer. In the "normal" sample B0 [see Fig. 8(a)], a 5-nm $\mathrm{In}_{0.75} \mathrm{Al}_{0.25} \mathrm{As}$ spacer followed by the Mn-doping layer was grown after the InAs/InGaAs channel, so that the InAs QW is free of $\mathrm{Mn}^{42,43}$ In this sample, the InAs QW is located $2.5 \mathrm{~nm}$ away from the channel border, it is facing the $\mathrm{Mn}$ site, and is separated from Mn layer by $7.5 \mathrm{~nm}$. The hole density and mobility obtained by magnetotransport measurements are $n_{h}=5.1 \times 10^{11} \mathrm{~cm}^{-2}$ and $\mu=8.6 \times$ $10^{3} \mathrm{~cm}^{2} /$ Vs. In the "inverted" doped structures B1 [see Fig. 8(b)], the Mn-doped layer is also separated from the InAs QW by $7.5 \mathrm{~nm}$, but is deposited before the channel growth. Due to segregation, this growth leads to a significant concentration of Mn ions in the InAs QW. The hole density in this sample is $n_{h}=4.4 \times 10^{11} \mathrm{~cm}^{-2}$ and the mobility is reduced by at least a factor of 2 compared to sample B0.

Figures 9 and 10 show the magnetic field and temperature dependencies of the photocurrent generated in sample B1 under low-power $\mathrm{THz}$ and $\mathrm{mw}$ excitation. These data reveal that the temperature decrease leads to a drastic enhancement of the photocurrent magnitude as well as it changes the linear in $B$ dependence of the signal into a Brillouin-function-like saturation. ${ }^{44}$ These results, which are similar to those obtained in $n$-type $(\mathrm{Cd}, \mathrm{Mn}) \mathrm{Te}$ DMS samples, are well described by Eqs. (2), (3), and (5), and provide a clear evidence for the exchange-interaction-based origin of the observed photocurrent. The inset in Fig. 10 demonstrates that for mw excitation, raising temperature does not result in the inversion of the current direction. This behavior is expected for $p$-type InAs DMS structures, in which, in contrast to $n$-type II-VI QWs, the intrinsic $g_{h}$ factor for carriers and the exchange integral have the same sign. For terahertz excitation, a tiny positive

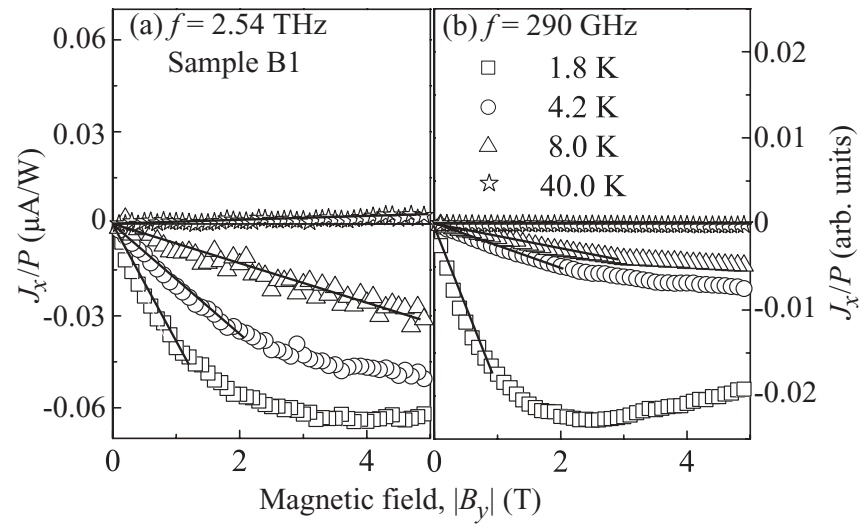

FIG. 9. Magnetic field dependence of photocurrent in inverted Mn-doped (In,Ga)As/InAlAs DMS QW at various temperatures. (a) and (b) show photocurrent induced by $\mathrm{THz}$ radiation, $f=$ $2.54 \mathrm{THz}$, and mw radiation, $f=290 \mathrm{GHz}$, respectively. Solid lines are linear fits for low $B$ as guides for the eye.

photocurrent is observed for $T \gtrsim 40 \mathrm{~K}$, which we attribute to the interplay of the negative intrinsic spin photocurrent and positive orbital photocurrent. ${ }^{24}$ Orbital photocurrent may also be responsible for a weaker temperature dependence of the THz-radiation-induced photocurrent compared to the one excited by $\mathrm{mw}$ radiation. The photocurrent excited in the normal Mn-doped sample B0, by contrast, is vanishingly small and we do not observe any substantial increase of its magnitude upon sample cooling. For both samples, the signal is almost independent of the orientation of the radiation plane. This observation demonstrates that the photocurrent is dominated by the relaxation mechanism.

Experiments applying high-power pulsed $\mathrm{THz}$ laser radiation to both B0 and B1 samples reveal that, similarly to the data obtained for $n$-type (Cd,Mn)Te DMS samples [Fig. 7(a)], at all temperatures the signal linearly increases with raising magnetic field. Also, the temperature dependence is very similar to that detected in $n$-type (Cd,Mn)Te DMS samples [Fig. 7(b)], demonstrating that at high power the exchange interaction is substantially reduced by the heating

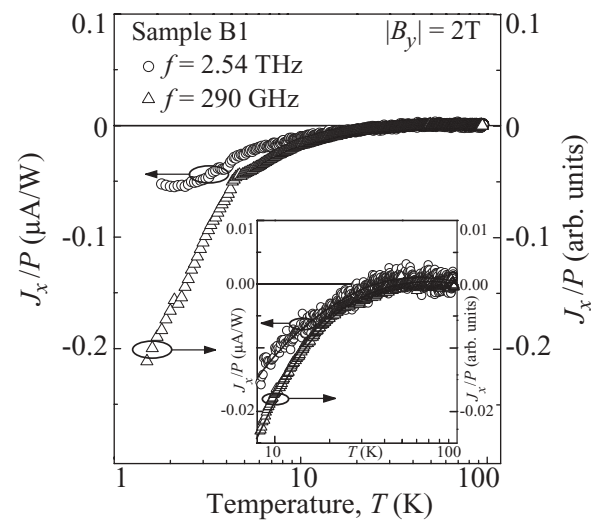

FIG. 10. Temperature dependence of photocurrent in $p$-doped (In,Ga)As/InAlAs:Mn DMS QW obtained at fixed magnetic field $\left|B_{y}\right|=2 \mathrm{~T}$ applying mw, $f=290 \mathrm{GHz}$, and THz, $f=2.54 \mathrm{THz}$, radiation. The inset shows a zoom of the high-temperature range with solid lines as guides for the eye. 
of the manganese system. The same results are obtained for the nonmagnetic reference $n$-type InAs QW sample doped by $\mathrm{Si}$ excited by high-power $\mathrm{THz}$ light as well by low-power $\mathrm{THz}$ and mw radiation. All these observations in III-V based structures excited by high-power radiation are in a good agreement with the theory of spin-polarized photocurrents in nonmagnetic semiconductor structures (see Sec. II).

While it was clearly observed in sample B1 at low-power excitation, at first glance, the Mn doping outside of the conducting channel should not result in a magnetic behavior because the wave function of the carrier does not penetrate to the Mn location. However, the B1 sample is doped on the substrate side and the $\mathrm{Mn}$ atoms penetrate towards the conducting channel due to segregation of $\mathrm{Mn}$ atoms during the structure growth. The segregation results in the presence of $\mathrm{Mn}^{2+}$ ions in the vicinity of the two-dimensional hole gas. The enhanced magnetic properties manifest themselves by the colossal negative magnetoresistance and the associated field-induced insulator-to-metal transition observed in such structures. ${ }^{45}$ By contrast, in the $p$-type InAs QW sample with Mn doping on the surface side (sample B0), the segregation shifts the Mn atom distribution away from the two-dimensional (2D) channel and the giant Zeeman splitting of the hole subbands in InAs QWs is almost absent. The absence of the giant Zeeman splitting in sample B0 substantiates the absence of residual $\mathrm{Mn}^{2+}$ ions in close vicinity to the two-dimensional hole gas. This further verifies the interpretation of earlier magnetotransport experiments. ${ }^{41}$

Similarly to II-VI DMS samples A1 and A2, we observed that in the inverted sample B1 the magnitude of the photocurrent measured at $1.8 \mathrm{~K}$ is about two orders of magnitude larger than that at $40 \mathrm{~K}$. Such enhancement is larger than that expected for the giant Zeeman spin splitting and provides an indirect evidence for the substantial contribution of the photocurrent due to scattering by magnetic ions. However, the direct comparison of the current variation to the Zeeman spin splitting is impossible because no PL or time-resolved Kerr rotation data for the InAs-based QWs are in our disposal.

\section{Heterovalent $\boldsymbol{n}$-AISb/InAs/ZnMnTe quantum wells}

InAs-based DMS structures are usually characterized by $p$-type conductivity. ${ }^{41,46}$ Concerning $n$-type In(Mn)As DMS, only thin films and superlattices with mobilities in the order of 100 to $1000 \mathrm{~cm}^{2} /$ Vs have been reported so far. ${ }^{4-50}$ The realization of $n$-type InAs-based DMS QWs with high mobility and controllable exchange interaction remains an important issue. A possible way to achieve this goal is to extend the heterovalent growth technology by the doping with magnetic ions. While III-V and II-VI DMS systems are widely studied and their magnetic properties are well known, heterovalent $n$-type AlSb/InAs/ZnMnTe quantum wells are new in the DMS family. These structures combine a narrow-gap III-V QW with wide-gap II-VI barriers. ${ }^{51}$ Manganese is introduced into the ZnTe barrier where it substitutes $\mathrm{Zn}$ and keeps electrically neutral providing a localized spin $S=5 / 2$. The enhanced magnetic properties are caused by the penetration of electron wave function of two-dimensional electrons into the
$(\mathrm{Zn}, \mathrm{Mn}) \mathrm{Te}$ layer and can be controllably varied by the position and concentration of $\mathrm{Mn}^{2+}$ ions. ${ }^{52}$

To fabricate $\mathrm{AlSb} / \mathrm{InAs} /(\mathrm{Zn}, \mathrm{Mn}) \mathrm{Te}$ heterovalent structures with Mn-containing barriers, two separate molecular-beam epitaxy (MBE) chambers have been applied, one for the III-V and the other for the II-VI part. In the III-V MBE machine, a buffer layer of $\mathrm{GaSb}$ containing a strained $\mathrm{AlSb} / \mathrm{GaSb}$ superlattice was grown. It follows by a 4-nm-thick AlSb barrier and a 15-nm-thick InAs QW.

Before the first III-V part was transferred to the II-VI MBE setup, the structure was passivated ex situ by sulfur exchanging a surface oxide, which then could be easily removed to start a coherent growth of ZnTe on top of InAs. In order to obtain a diluted magnetic semiconductor barrier of InAs QW, sample $\mathrm{C} 1$, a $1-\mathrm{ML}(\approx 0.32 \mathrm{~nm}) \mathrm{MnTe}$ was introduced into the $\mathrm{ZnTe}$ barrier at a $10-\mathrm{ML}$ distance from the QW. By that, as a result of the segregation and diffusion processes, we obtain structure with Mn ions distributed over several monolayers of the surrounding $\mathrm{ZnTe}$. The maximum content of the remaining $\mathrm{MnTe}$ is estimated to be well below $30 \mathrm{~mol} \%$. Structure C 2 has the same spacer with an adjacent $10-\mathrm{nm} \mathrm{Zn}_{0.9} \mathrm{Mn}_{0.1}$ Te layer of lower Mn concentration per ML. Sample C0 is a reference structure with nonmagnetic ZnTe barrier.

The two-dimensional electron gas has the density $n_{e} \sim$ $(1 \div 2) \times 10^{13} \mathrm{~cm}^{-2}$ and the mobility $\mu \sim 5 \times 10^{3} \mathrm{~cm}^{2} / \mathrm{Vs}$ at $T=4.2 \mathrm{~K}$. The most of $2 \mathrm{D}$ electrons in hybrid $\mathrm{QW}$ originate from donor centers located at III-V/II-VI heterovalent interface resulting in the large surface density of positively charged donor centers at the interface, while the Fermi level within the InAs layer is pinned to that in the GaSb and ZnTe layers. Consequently, the structures become highly asymmetric due to a strong built-in electric field. The band structure of the sample C1 is sketched in Fig. 11(a).

The magnetic field and temperature dependencies of the photocurrent induced in the DMS sample $\mathrm{C} 1$ are shown in Figs. 12 and 13(a), respectively. Both plots demonstrate the characteristic influence of $\mathrm{Mn}^{2+}$ ions aligned by the external magnetic field. The sign inversion of the photocurrent and the strong enhancement of its magnitude by cooling the sample as well as the nonlinear magnetic field behavior (saturation at high $B$ ) are clearly observed. ${ }^{53}$ The picture remains qualitatively the same for both low-power $\mathrm{THz}$
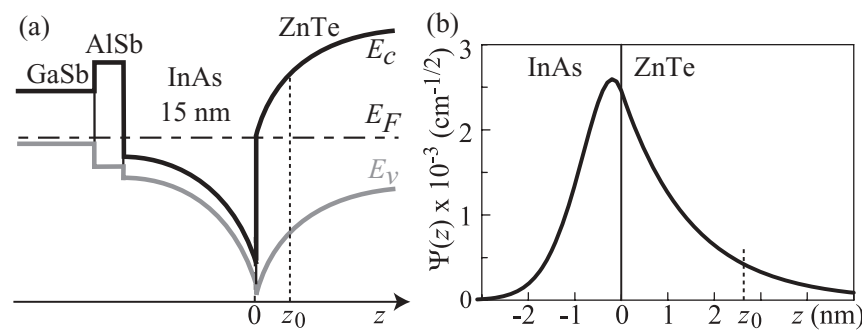

FIG. 11. (a) Sketch of the band structure of hybrid $\mathrm{AlSb} / \mathrm{InAs} / \mathrm{ZnTe}$ samples; dotted line indicates the position of MnTe layer in sample $\mathrm{C} 1$. (b) Electron wave function $\Psi(z)$ calculated for a triangular QW with the QW potential gradient $1.8 \times 10^{7} \mathrm{eV} / \mathrm{cm}$ resulted from ionized donors at interface with the density $2 \times$ $10^{13} \mathrm{~cm}^{-2}$, flat barriers, and the effective mass $m^{*}=0.1 m_{0}$. The latter corresponds to $m^{*}$ at conduction band bottom in ZnTe as well as in InAs with nonparabolicity being taken into account. 


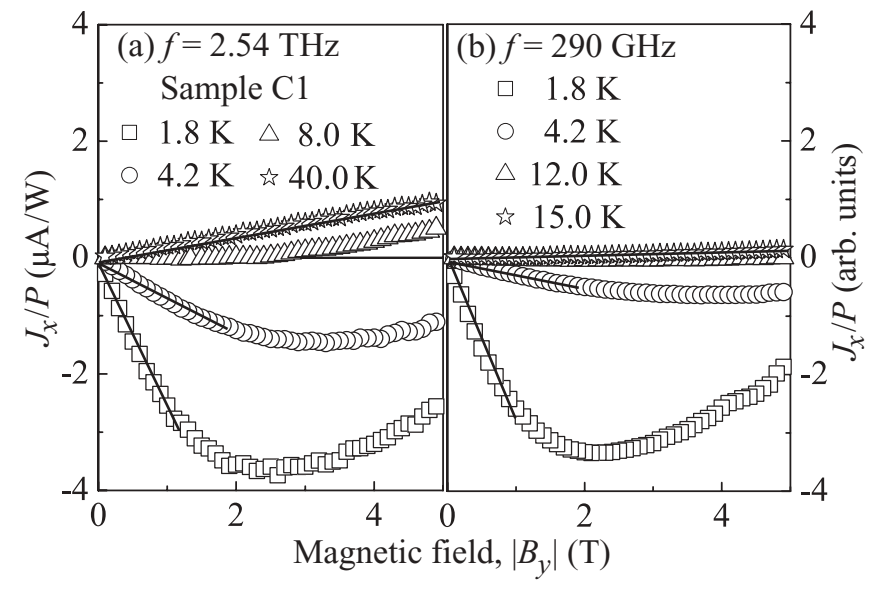

FIG. 12. Magnetic field dependence of photocurrent measured in hybrid sample $\mathrm{C} 1$ at various temperatures and applying (a) $\mathrm{THz}$ radiation, $f=2.54 \mathrm{THz}$, and (b) mw radiation, $f=290 \mathrm{GHz}$. Solid lines are linear fits for low $B$ as guides for the eye.

and mw radiations. The only difference is the value of the inversion temperature, which is about $15 \mathrm{~K}$ for mw-radiationinduced photocurrent and about $9 \mathrm{~K}$ for $\mathrm{THz}$ photocurrent. Figure 13 also shows the data for the reference nonmagnetic $\mathrm{AlSb} / \mathrm{InAs} / \mathrm{ZnTe} \mathrm{QW}$ sample C0. Here, in contrast to the sample $\mathrm{C} 1$, the photocurrent shows linear dependence on the external magnetic field in the whole temperature range, it does not depend substantially on temperature for $T<30 \mathrm{~K}$, and for $T>30 \mathrm{~K}$ decreases as $J \propto 1 / T$. In sample $\mathrm{C} 2$ with $\mathrm{Zn}_{0.9} \mathrm{Mn}_{0.1} \mathrm{Te}$ inserted in the barrier and distributed over a larger distance from QW, we observed less pronounced DMS properties (not shown). The photocurrent changes its sign upon cooling the samples at $T \approx 2.5 \mathrm{~K}$, but at low temperature its magnitude is substantially lower than that detected in sample C1.

All these findings give a strong evidence for a substantial influence of the exchange coupling between the 2D electrons and the $\mathrm{Mn}$ atoms introduced in the $\mathrm{ZnTe}$ barrier in sample $\mathrm{C} 1$ and less pronounced effect of magnetic impurities in sample
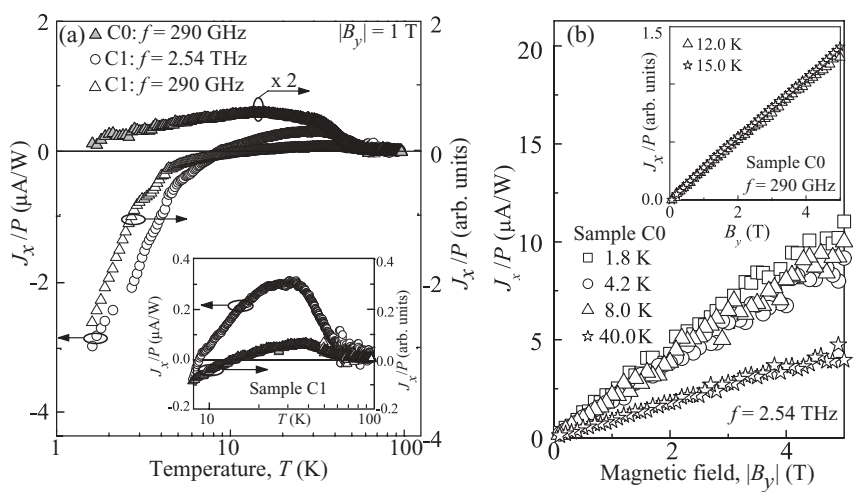

FIG. 13. (a) Temperature dependence of photocurrent measured in samples $\mathrm{C} 1$ and $\mathrm{C} 0$ at fixed magnetic field $\left|B_{y}\right|=1 \mathrm{~T}$ applying mw radiation, $f=290 \mathrm{GHz}$, and $\mathrm{THz}$ radiation, $f=2.54 \mathrm{THz}$. The inset shows a zoom of the high-temperature region. (b) Magnetic field dependence of photocurrent excited in hybrid nonmagnetic reference sample $\mathrm{C} 0$ at various temperatures applying $\mathrm{THz}, f=2.54 \mathrm{THz}$, and (in inset) mw radiation.
C2. In both magnetic samples $\mathrm{C} 1$ and $\mathrm{C} 2$ (Fig. 11), the $\mathrm{Mn}$ layers are separated from the QW by 10-ML-thick spacer. Therefore, the exchange interaction is supposed to be mediated via a penetration of the electron wave function $\Psi(z)$ into the barrier. ${ }^{54}$ The Zeeman splitting in structures with $\mathrm{Mn}$ ions $\delta$ layer placed at $z=z_{0}$ can be estimated using the standard expression

$$
E_{\mathrm{Z}}=g_{e} \mu_{\mathrm{B}} B+\alpha_{e} N_{\mathrm{Mn}}\left|\Psi\left(z_{0}\right)\right|^{2} S_{0} \mathrm{~B}_{5 / 2}\left(\frac{5 \mu_{\mathrm{B}} g_{\mathrm{Mn}} B}{2 k_{\mathrm{B}}\left(T_{\mathrm{Mn}}+T_{0}\right)}\right),
$$

where $N_{\mathrm{Mn}}$ is the sheet Mn density. In contrast to Eq. (5), this equation is also valid for the DMS structures where Mn ions are introduced into the barrier. The necessary overlap of the wave function with the ions is ensured by the strong asymmetry of the QWs due to the built-in electric field discussed above. The calculations prove that the wave function deeply penetrates into ZnTe resulting in the substantial overlap of $\Psi(z)$ and $\mathrm{Mn}^{2+}$ ions in C1 structure [see Fig. 11(b)]. Due to the opposite signs of $g_{e}$ in InAs and $\alpha_{e} N_{\mathrm{Mn}}$, under sample cooling the sign of $E_{\mathrm{Z}}$ inverses, resulting in the reversion of the photocurrent direction as observed for the $\mathrm{C} 1$ structure (see Figs. 12 and 13), and as well as for the C2 sample. In sample $\mathrm{C} 1$ with $\mathrm{Mn} \delta$ layer, the current behavior at low temperature is dominated by the exchange interaction and almost follows the Brillouin function: it is amplified by cooling the sample and, at low temperatures, saturates with raising magnetic field [see Figs. 12 and 13(a)]. Estimations of the Zeeman spin splitting in sample C1 made after Eq. (10) using $N_{\mathrm{Mn}}=10^{15} \mathrm{~cm}^{-2}$ and $\alpha_{e}=10^{-20} \mathrm{meV} \mathrm{cm}^{3}$ (see Ref. 55) show that at $T=1.8 \mathrm{~K}$ and $B=2 \mathrm{~T}$ exchange spin splitting should be one order of magnitude larger than the intrinsic Zeeman splitting. This estimated value agrees well with experimental findings [see Fig. 13(a)], and indicates that the Zeeman splitting based mechanism dominates the current formation.

The photocurrent data obtained on sample $\mathrm{C} 2$ show much less pronounced magnetic properties and give a further support of the suggested mechanism for exchange interaction in C-type DMS structures. Indeed, because of spatial distribution of the Mn over larger distance from QW, in sample C2 the overlap of the electron wave function with the $\mathrm{Mn}^{2+}$ ions should be substantially smaller than that in the $\mathrm{C} 1$ structure.

\section{SUMMARY}

In summary, we demonstrate that the irradiation of various types of low-dimensional diluted magnetic semiconductors by low-power terahertz or mw radiation causes spin-polarized electric current if an in-plane magnetic field is applied. Microscopically, the effect originates from the spin-dependent asymmetric scattering of carriers resulting in a pure spin current which is converted into a spin-polarized electric current by magnetic field. Furthermore, its behavior clearly reflects all characteristic features of the exchange interaction and is giantly enhanced at low temperatures. The spin-polarized electric current enhancement is caused by the exchange interaction of carriers with $\mathrm{Mn}^{2+}$ ions resulting in the giant Zeeman splitting. In the structures with the $\mathrm{Mn}^{2+}$ ions in the quantum well, the efficiency of the current generation is additionally amplified due to the spin-dependent scattering of 
carriers by polarized $\mathrm{Mn}^{2+}$ ions. Our measurements carried out on II-VI, III-V, and hybrid II-V/II-VI QW structures doped by Mn demonstrate that the effect is very general and can be used for the efficient generation of spin-polarized electric currents, e.g., applying conventional Gunn diodes, as well as for the study of DMS materials. The latter could be of particular importance for exploring DMS properties in materials hardly accessible by optical or transport measurements. As for the future tasks, experiments providing a direct evidence for spin polarization of the photocurrent, e.g., by measurements of the spin Kerr effect, would give a deeper insight in the origin and microscopic mechanisms of this phenomenon.

\section{ACKNOWLEDGMENTS}

The financial support from the DFG (SFB 689), the Linkage Grant of IB of BMBF at DLR, RFBR, RF President Grant MD-2062.2012.2, and the Foundation "Dynasty" is gratefully acknowledged. The research in Poland was partially supported by the EU within European Regional Development Fund, through grant Innovate Economy (Grant No. POIG.01.01.0200-008/08).

\section{APPENDIX: CALCULATION OF ELECTRON FLUXES IN THE SPIN SUBBANDS}

Here, we derive analytical equations for the electron fluxes $\boldsymbol{i}_{ \pm 1 / 2}$ in the spin subbands. We consider the excitation mechanism which is responsible for polarization-dependent spin current. In the framework of kinetic theory, the fluxes are given by

$$
i_{s}=\sum_{k} \boldsymbol{v}_{k} f_{s k}
$$

where $s= \pm 1 / 2, \boldsymbol{v}_{\boldsymbol{k}}=\hbar \boldsymbol{k} / m^{*}$ is the electron velocity, $m^{*}$ is the effective mass, and $f_{s k}$ is the electron distribution functions in the spin subband.

\section{Quasiclassical theory}

For low-frequency electromagnetic field $\omega \ll \bar{E} / \hbar$, the distribution functions of carriers in the spin subbands can be found from the Boltzmann equation

$$
\frac{\partial f_{s \boldsymbol{k}}}{\partial t}+e \boldsymbol{E}(t) \frac{\partial f_{s \boldsymbol{k}}}{\hbar \partial \boldsymbol{k}}=\operatorname{St} f_{s \boldsymbol{k}},
$$

where $\boldsymbol{E}(t)=\boldsymbol{E} \exp (-i \omega t)+\boldsymbol{E}^{*} \exp (+i \omega t)$ is the electric field of radiation in QW, $\boldsymbol{E}$ is the (complex) field amplitude, and $\mathrm{St} f_{s \boldsymbol{k}}$ is the collision integral. In the case of spinconserving elastic scattering of electrons by impurities or structure defects, the collision integral has the form

$$
\mathrm{St} f_{s \boldsymbol{k}}=\sum_{\boldsymbol{k}^{\prime}}\left(W_{s \boldsymbol{k}, \boldsymbol{s} \boldsymbol{k}^{\prime}} f_{s \boldsymbol{k}^{\prime}}-W_{s \boldsymbol{k}^{\prime}, s \boldsymbol{k}} f_{s \boldsymbol{k}}\right),
$$

where $W_{s \boldsymbol{k}, \boldsymbol{s} \boldsymbol{k}^{\prime}}=(2 \pi / \hbar)\left\langle\left|V_{s \boldsymbol{k}, \boldsymbol{s} \boldsymbol{k}^{\prime}}\right|^{2}\right\rangle \delta\left(\varepsilon_{\boldsymbol{k}}-\varepsilon_{\boldsymbol{k}^{\prime}}\right)$ is the scattering rate in the spin subband, $V_{s \boldsymbol{k}, \boldsymbol{s} \boldsymbol{k}^{\prime}}$ is the matrix element of scattering, $\varepsilon_{\boldsymbol{k}}=\hbar^{2} \boldsymbol{k}^{2} /\left(2 m^{*}\right)$, and the angle brackets denote averaging over scatterers. Taking into account $\boldsymbol{k}$-linear contributions to the matrix element of scattering [Eq. (1)], one obtains, e.g., for the spin projections $\pm 1 / 2$ onto the $y$ axis,

$$
\left\langle\left|V_{s \boldsymbol{k}, s \boldsymbol{k}^{\prime}}\right|^{2}\right\rangle=\left\langle V_{0}^{2}\right\rangle+4 s\left\langle V_{0} V_{y x}\right\rangle\left(k_{x}+k_{x}^{\prime}\right) .
$$

It is assumed in Eq. (A4) that the matrix elements $V_{0}$ and $V_{y x}$ are real, $\left|V_{y x}\right| \ll\left|V_{0}\right|$, and $\left\langle V_{0} V_{y x}\right\rangle$ and $\left\langle V_{0} V_{x y}\right\rangle$ are the only nonzero components of the tensor $\left\langle V_{0} V_{\alpha \beta}\right\rangle$ in (001)grown QWs. In the following, we suggest that the scattering asymmetry is caused by short-range impurities or defects and, therefore, $\left\langle V_{0} V_{y x}\right\rangle$ is independent of the directions of the wave vectors $\boldsymbol{k}$ and $\boldsymbol{k}^{\prime}$.

To solve the kinetic Eq. (A2), we expand the distribution functions in series of powers of the electric field

$$
f_{s k}=f_{s}^{(0)}(\varepsilon)+\left[f_{s k}^{(1)} e^{-i \omega t}+\text { c.c }\right]+f_{s k}^{(2)}+\cdots,
$$

where $f_{s}^{(0)}(\varepsilon)$ is the equilibrium distribution function of electrons in the spin subband, $f_{s \boldsymbol{k}}^{(1)} \propto|\boldsymbol{E}|$, and $f_{s \boldsymbol{k}}^{(2)} \propto|\boldsymbol{E}|^{2}$. The first-order corrections to the equilibrium distribution function oscillate at the radiation field frequency $\omega$ and do not contribute to dc fluxes. The directed fluxes $\boldsymbol{i}_{ \pm 1 / 2}$ in the spin subbands are determined by the second order in $\boldsymbol{E}$ corrections and obtained by multiplying $f_{s k}^{(2)}$ by the velocity and summing up the result over the momentum [see Eq. (A1)]. Such a procedure yields

$$
\begin{aligned}
& i_{s, x}=M_{1, s}\left(\left|E_{x}\right|^{2}-\left|E_{y}\right|^{2}\right)+M_{2, s}|\boldsymbol{E}|^{2}, \\
& i_{s, y}=M_{1, s}\left(E_{x} E_{y}^{*}+E_{y} E_{x}^{*}\right)+M_{3, s} i\left(\boldsymbol{E} \times \boldsymbol{E}^{*}\right)_{z},
\end{aligned}
$$

where

$$
\begin{gathered}
M_{1, s}=\frac{4 s e^{2}\left\langle V_{0} V_{y x}\right\rangle}{\hbar^{4}} \sum_{k} \frac{\tau_{p} d\left(\tau_{p} \tau_{2} \varepsilon^{2}\right) / d \varepsilon}{1+\left(\omega \tau_{p}\right)^{2}} \frac{d f_{s}^{(0)}}{d \varepsilon}, \\
M_{2, s}=\frac{4 s e^{2}\left\langle V_{s} V_{y x}\right\rangle}{\hbar^{4}} \sum_{k} \frac{\left(1-\omega^{2} \tau_{p} \tau_{2}\right) \tau_{p} \tau_{2} \varepsilon^{2} \tau_{p}^{\prime}}{\left[1+\left(\omega \tau_{p}\right)^{2}\right]\left[1+\left(\omega \tau_{2}\right)^{2}\right]} \frac{d f_{s}^{(0)}}{d \varepsilon},
\end{gathered}
$$

$$
M_{3, s}=-\frac{4 s e^{2}\left\langle V_{s} V_{y x}\right\rangle}{\hbar^{4}} \sum_{k} \frac{\omega \tau_{p} \tau_{2}\left(\tau_{p}+\tau_{2}\right) \varepsilon^{2} \tau_{p}^{\prime}}{\left[1+\left(\omega \tau_{p}\right)^{2}\right]\left[1+\left(\omega \tau_{2}\right)^{2}\right]} \frac{d f_{s}^{(0)}}{d \varepsilon}
$$

where $\tau_{p}$ and $\tau_{2}$ are the relaxation times of the first and second angular harmonics of the distribution function,

$$
\begin{aligned}
\tau_{p}^{-1} & =(2 \pi / \hbar) \sum_{\boldsymbol{k}^{\prime}}\left\langle V_{0}^{2}\right\rangle\left(1-\cos \theta_{\boldsymbol{k} \boldsymbol{k}^{\prime}}\right) \delta\left(\varepsilon_{\boldsymbol{k}}-\varepsilon_{\boldsymbol{k}^{\prime}}\right), \\
\tau_{2}^{-1} & =(2 \pi / \hbar) \sum_{\boldsymbol{k}^{\prime}}\left\langle V_{0}^{2}\right\rangle\left(1-\cos 2 \theta_{\boldsymbol{k} \boldsymbol{k}^{\prime}}\right) \delta\left(\varepsilon_{\boldsymbol{k}}-\varepsilon_{\boldsymbol{k}^{\prime}}\right),
\end{aligned}
$$

$\theta_{\boldsymbol{k} \boldsymbol{k}^{\prime}}$ is the angle between $\boldsymbol{k}$ and $\boldsymbol{k}^{\prime}$, and $\tau_{p}^{\prime}=d \tau_{p} / \varepsilon$. The fluxes $\boldsymbol{i}_{+1 / 2}$ and $\boldsymbol{i}_{-1 / 2}$ are directed oppositely to each other, forming a pure spin current for equal distribution functions in the spin subbands $f_{ \pm 1 / 2}^{(0)}$ and equal scattering rates.

It follows from Eqs. (A6)-(A9) that the fluxes $\boldsymbol{i}_{ \pm 1 / 2}$ depend on the radiation polarization state. For linearly polarized radiation, the dependence of the $x$ and $y$ components on the azimuth angle $\beta$ is given by Eq. (4) because $\left|E_{x}\right|^{2}-$ $\left|E_{y}\right|^{2}=-|\boldsymbol{E}|^{2} \cos 2 \beta$ and $E_{x} E_{y}^{*}+E_{y} E_{x}^{*}=-|\boldsymbol{E}|^{2} \sin 2 \beta$ for the experimental geometry used. The term proportional to $M_{3, s}$ in Eq. (A6) describes the contribution to spin current that is sensitive to the sign of radiation helicity. It can be excited by circularly or, in general, elliptically polarized radiation 
and reversed by changing the sign of circular polarization. Equation (A9) shows that the helicity-sensitive current in QWs emerges due to the energy dependence of the momentum relaxation time. If electrons are scattered by short-range impurities or defects, then the energy dependence of $\tau_{p}$ and $\tau_{2}$ can be neglected, $\tau_{p}=\tau_{2}$, and the parameter $M_{1, s}$ takes the form

$$
M_{1, s}=-8 \frac{s n_{s}}{m^{*} \hbar} \frac{\tau_{p}^{2} e^{2}}{1+\left(\omega \tau_{p}\right)^{2}} \frac{\left\langle V_{0} V_{y x}\right\rangle}{\left\langle V_{0}^{2}\right\rangle},
$$

while $M_{2, s}$ and $M_{3, s}$ vanish. In this case, the excitation mechanism leads only to the current contribution which is sensitive to the linear polarization of radiation. The polarization-independent fluxes in the spin subbands are completely determined by the energy relaxation mechanism.

\section{Quantum theory}

The presented above quasiclassical approach is not valid if the photon energy $\hbar \omega$ is comparable or exceeds $\bar{E}$. In this spectral range, an adequate microscopic theory of spincurrent generation can be developed in the framework of quantum consideration of intrasubband optical transitions in QW. Such transitions are accompanied by electron scattering by impurities, acoustic or optical phonons, etc., because of the need for energy and momentum conservation. To first order in spin-orbit interaction, the matrix element of optical transitions in the subbands with the spin projection $s= \pm 1 / 2$ along $y$ accompanied by elastic electron scattering from short-range impurities has the form ${ }^{14,56}$

$$
M_{s \boldsymbol{k}, s \boldsymbol{k}^{\prime}}=\frac{e \boldsymbol{A} \cdot\left(\boldsymbol{k}-\boldsymbol{k}^{\prime}\right)}{c \omega m^{*}} V_{s \boldsymbol{k}, s \boldsymbol{k}^{\prime}}-4 s \frac{e A_{x}}{c \hbar} V_{y x},
$$

where $\boldsymbol{A}=-i(c / \omega) \boldsymbol{E}$ is the vector potential of the electromagnetic wave and $c$ is the speed of light. The first term on the right-hand side of Eq. (A11) describes transitions $\left(e 1, s, \boldsymbol{k}^{\prime}\right) \rightarrow$ $(e 1, s, \boldsymbol{k})$ with intermediate states in the conduction subband $e 1$, the second term corresponds to the transitions via intermediate states in other bands.

We assume that the radiation frequency is high enough, $\omega \tau_{p} \gg 1$. Then, the distribution functions of electrons in the spin subbands satisfy the kinetic equation

$$
g_{s k}=\mathrm{St} f_{s k},
$$

where $g_{s k}$ is the optical generation rate,

$$
g_{s \boldsymbol{k}}=\frac{2 \pi}{\hbar} \sum_{\boldsymbol{k}^{\prime}, \pm}\left|M_{s \boldsymbol{k}, s \boldsymbol{k}^{\prime}}\right|^{2}\left(f_{s \boldsymbol{k}^{\prime}}-f_{s \boldsymbol{k}}\right)\left(\varepsilon_{\boldsymbol{k}}-\varepsilon_{\boldsymbol{k}^{\prime}} \pm \hbar \omega\right),
$$

and $\mathrm{St} f_{s k}$ is the collision integral given by Eq. (A3). Taking into account spin-dependent terms in the generation rate and the collision integral, one can calculate the distribution functions $f_{s \boldsymbol{k}}$ and then, using Eq. (A1), the fluxes $\boldsymbol{i}_{s}$. Such a calculation yields

$$
\begin{aligned}
& i_{s, x}=-4 \frac{s n_{s} \kappa_{s} e^{2}}{\hbar m^{*} \omega^{2}} \frac{\left\langle V_{0} V_{y x}\right\rangle}{\left\langle V_{0}^{2}\right\rangle}\left(\left|E_{x}\right|^{2}-\left|E_{y}\right|^{2}\right), \\
& i_{s, y}=-4 \frac{s n_{s} \kappa_{s} e^{2}}{\hbar m^{*} \omega^{2}} \frac{\left\langle V_{0} V_{y x}\right\rangle}{\left\langle V_{0}^{2}\right\rangle}\left(E_{x} E_{y}^{*}+E_{y} E_{x}^{*}\right),
\end{aligned}
$$

where $\kappa_{s}$ is a dimensionless parameter that depends on the carrier distribution,

$$
\kappa_{s}=\frac{\int_{0}^{\infty}(1+2 \varepsilon / \hbar \omega)\left[f_{s}^{(0)}(\varepsilon)-f_{s}^{(0)}(\varepsilon+\hbar \omega)\right] d \varepsilon}{\int_{0}^{\infty} f_{s}^{(0)}(\varepsilon) d \varepsilon},
$$

and is equal to 1 and 2 for the cases $\hbar \omega \gg \bar{E}$ and $\hbar \omega \ll \bar{E}$, respectively. We note that, in the frequency range $1 / \tau_{p} \ll \omega \ll$ $\bar{E} / \hbar$, both the quasiclassical theory [Eqs. (A6) and (A10)] and the quantum theory [Eq. (A14)] give the same result.
${ }^{1}$ S. Maekawa, Concepts in Spin Electronics (Oxford University Press, Oxford 2006).

${ }^{2}$ J. Fabian, A. Matos-Abiague, C. Ertler, P. Stano, and I. Zutic, Acta Phys. Slovaca 57, 565 (2007).

${ }^{3}$ Spin Physics in Semiconductors, edited by M. I. Dyakonov (Springer, Berlin 2008).

${ }^{4}$ Spintronics, Semiconductors and Semimetals, Vol. 82, edited by T. Dietl, D. D. Awschalom, M. Kaminska, and H. Ohno, (Elsevier, Amsterdam 2008).

${ }^{5}$ M. W. Wu, J. H. Jiang, and M. Q. Weng, Phys. Rep. 493, 61 (2010). ${ }^{6}$ Semiconductor Spintronics and Quantum Computation edited by D. D. Awschalom, D. Loss, and N. Samarth (Springer, Berlin 2010).

${ }^{7}$ Handbook of Spin Transport and Magnetism, edited by E. Y. Tsymbal and I. Zutic (CRC Press, Boca Raton, FL, 2012).

${ }^{8}$ Introduction to the Physics of Diluted Magnetic Semiconductors, edited by J. Kossut and J. A. Gaj (Springer, Berlin 2010).

${ }^{9}$ Handbook of Spintronic Semiconductors, edited by W. M. Chen and I. A. Buyanova (Pan Stanford, Singapore, 2010).

${ }^{10}$ H. Ohno, Nat. Mater. 9, 952 (2010).

${ }^{11}$ T. Dietl, Nat. Mater. 9, 965 (2010).

${ }^{12}$ M. Flatte, Nat. Phys. 7, 285 (2011).
${ }^{13}$ J. K. Furdyna, J. Appl. Phys. 64, R29 (1988).

${ }^{14}$ S. D. Ganichev, V. V. Bel'kov, S. A. Tarasenko, S. N. Danilov, S. Giglberger, Ch. Hoffmann, E. L. Ivchenko, D. Weiss, W. Wegscheider, Ch. Gerl, D. Schuh, J. Stahl, J. De Boeck, G. Borghs, and W. Prettl, Nat. Phys. 2, 609 (2006)

${ }^{15}$ S. D. Ganichev, S. N. Danilov, V. V. Bel'kov, S. Giglberger, S. A. Tarasenko, E. L. Ivchenko, D. Weiss, W. Jantsch, F. Schäffler, D. Gruber, and W. Prettl, Phys. Rev. B 75, 155317 (2007).

${ }^{16}$ T. Yokoyama, Y. Tanaka, and N. Nagaosa, Phys. Rev. Lett. 106, 246601 (2011).

${ }^{17}$ S. D. Ganichev, S. A. Tarasenko, V. V. Bel'kov, P. Olbrich, W. Eder, D. R. Yakovlev, V. Kolkovsky, W. Zaleszczyk, G. Karczewski, T. Wojtowicz, and D. Weiss, Phys. Rev. Lett. 102, 156602 (2009).

${ }^{18}$ E. L. Ivchenko and S. A. Tarasenko, Semicond. Sci. Technol. 23, 114007 (2008).

${ }^{19}$ N. S. Averkiev, L. E. Golub, and M. Willander, J. Phys.: Condens. Matter 14, R271 (2002)

${ }^{20}$ E. L. Ivchenko and S. A. Tarasenko, Zh. Eksp. Teor. Fiz. 126, 426 (2004) [JETP 99, 379 (2004)].

${ }^{21}$ T. Wojtowicz, M. Kutrowski, G. Karczewski, J. Kossut, F. J. Teran, and M. Potemski, Phys. Rev. B 59, 10437 (1999). 
${ }^{22}$ J. C. Egues and J. W. Wilkins, Phys. Rev. 58, R16012 (1998).

${ }^{23}$ Since the average electron spin due to the giant Zeeman effect is parallel to $\boldsymbol{S}_{\mathrm{Mn}}$, the directions of currents $\boldsymbol{j}_{Z}$ and $\boldsymbol{j}_{S c}$ caused by the relaxation mechanism coincide for $u>0$.

${ }^{24}$ S. A. Tarasenko, Phys. Rev. B 77, 085328 (2008).

${ }^{25}$ S. A. Tarasenko, Phys. Rev. B 83, 035313 (2011).

${ }^{26}$ S. D. Ganichev, E. L. Ivchenko, and W. Prettl, Phys. E (Amsterdam) 14, 166 (2002).

${ }^{27}$ Z. D. Kvon, S. N. Danilov, N. N. Mikhailov, S. A. Dvoretsky, and S. D. Ganichev, Phys. E (Amsterdam) 40, 1885 (2008).

${ }^{28}$ S. D. Ganichev, Phys. B (Amsterdam) 273, 737 (1999); 274, 737 (1999).

${ }^{29}$ P. Schneider, J. Kainz, S. D. Ganichev, V. V. Bel'kov, S. N. Danilov, M. M. Glazov, L. E. Golub, U. Rössler, W. Wegscheider, D. Weiss, D. Schuh, and W. Prettl, J. Appl. Phys. 96, 420 (2004).

${ }^{30}$ C. Drexler, V. V. Bel'kov, B. Ashkinadze, P. Olbrich, C. Zoth, V. Lechner, Ya. V. Terent'ev, D. R. Yakovlev, G. Karczewski, T. Wojtowicz, D. Schuh, W. Wegscheider, and S. D. Ganichev, Appl. Phys. Lett. 97, 182107 (2010).

${ }^{31}$ S. A. Crooker, D. A. Tulchinsky, J. Levy, D. D. Awschalom, R. Garcia, and N. Samarth, Phys. Rev. Lett. 75, 505 (1995).

${ }^{32}$ J. Jaroszynski, T. Andrearczyk, G. Karczewski, J. Wróbel, T. Wojtowicz, E. Papis, E. Kaminska, A. Piotrowska, D. Popovic, and T. Dietl, Phys. Rev. Lett. 89, 266802 (2002).

${ }^{33}$ M. K. Kneip, D. R. Yakovlev, M. Bayer, G. Karczewski, T. Wojtowicz, and J. Kossut, Appl. Phys. Lett. 88, 152105 (2006).

${ }^{34}$ We note that in the theoretical parts of the paper, the current density $j$ is used while in experiment the electric current $J$ is measured, which is proportional to the current density.

${ }^{35}$ At low magnetic fields $B_{y} \lesssim 0.5 \mathrm{~T}$, a small deviation from the linear coupling between $J$ and $B$ is detected (see Fig. 4). The magnitude of this feature depends on the contacts quality and, most probably, originates from the illumination of contacts. However, this spurious signal is small compared to that at high magnetic fields and will not be discussed in the following.

${ }^{36}$ Note that the photocurrent pulses have typical duration of about $100 \mathrm{~ns}$, which corresponds to the terahertz laser-pulse length.

${ }^{37}$ An additional reason for this discrepancy could be a contribution of the orbital current (Refs. 24 and 25), which, as discussed in Sec. II, is almost insensitive to the enhanced magnetic properties and is out of scope of this paper.

${ }^{38}$ V. V. Bel'kov, P. Olbrich, S. A. Tarasenko, D. Schuh, W. Wegscheider, T. Korn, C. Schüller, D. Weiss, W. Prettl, and S. D. Ganichev, Phys. Rev. Lett. 100, 176806 (2008).
${ }^{39}$ M. Kohda, T. Kita, Y. Ohno, F. Matsukura, and H. Ohno, Appl. Phys. Lett. 89, 012103 (2006).

${ }^{40}$ T. Dietl, in Handbook on Semiconductors, Vol. 3b, edited by T. S. Moss (North-Holland, Amsterdam, 1994).

${ }^{41}$ U. Wurstbauer and W. Wegscheider, Phys. Rev. B 79, 155444 (2009).

${ }^{42}$ G. Prechtl, W. Heiss, A. Bonanni, W. Jantsch, S. Mackowski, and E. Janik, Phys. Rev. B 68, 165313 (2003).

${ }^{43}$ U. Wurstbauer, M. Soda, R. Jakiela, D. Schuh, D. Weiss, J. Zweck, and W. Wegscheider, J. Cryst. Growth 311, 2160 (2009).

${ }^{44} \mathrm{~A}$ small deviation from the saturation behavior seen for microwave data we attribute to the contribution of the orbital current discussed in Sec. II.

${ }^{45}$ U. Wurstbauer, C. Sliwa, D. Weiss, T. Dietl, and W. Wegscheider, Nat. Phys. 6, 955 (2010).

${ }^{46}$ H. Ohno, H. Munekata, T. Penney, S. von Molnar, and L. L. Chang, Phys. Rev. Lett. 68, 2664 (1992).

${ }^{47}$ H. Munekata, H. Ohno, S. von Molnar, A. Segmüller, L. L. Chang, and L. Esaki, Phys. Rev. Lett. 63, 1849 (1989).

${ }^{48}$ Y. L. Soo, S. W. Huang, Z. H. Ming, Y. H. Kao, H. Munekata, and L. L. Chang, Phys. Rev. B 53, 4905 (1996).

${ }^{49}$ G. Acbas, G. B. Kim, X. Chen, S. Wang, M. Cheon, C. J. Meining, H. Luo, B. D. McCombe, Y. Sasaki, X. Liu, and J. K. Furdyna, Phys. E (Amsterdam) 20, 382 (2004).

${ }^{50}$ G. A. Khodaparast, M. A. Zudov, J. Kono, Y. H. Matsuda, T. Ikaida, S. Ikeda, N. Miura, T. Slupinski, A. Oiwa, H. Munekata, G. D. Sanders, Y. Sun, and C. J. Stanton, J. Supercond. 16, 107 (2003).

${ }^{51}$ S. V. Ivanov, V. A. Kaygorodov, S. V. Sorokin, V. A. Solov'ev, A. A. Sitnikova, O. G. Lyublinskaya, Ya. V. Terent'ev, Yu. B. Vasilyev, V. L. Berkovits, A. A. Toropov, and P. S. Kop'ev, Phys. Status Solidi C 1, 1468 (2004).

${ }^{52}$ Ya. V. Terent'ev, C. Zoth, V. V. Bel'kov, P. Olbrich, C. Drexler, V. Lechner, P. Lutz, A. N. Semenov, V. A. Solov'ev, I. V. Sedova, G. V. Klimko, T. A. Komissarova, S. V. Ivanov, and S. D. Ganichev, Appl. Phys. Lett. 99, 072111 (2011).

${ }^{53} \mathrm{As}$ in the discussion of the results obtained for DMS samples of A and B types, we attribute a deviation from the saturation behavior observed for high magnetic fields to the contributions of intrinsic and orbital photocurrents.

${ }^{54}$ E. Z. Meilikhov and R. Z. Farzedinova, Zh. Eksp. Teor. Fiz. 137, 907 (2010) [JETP 110, 794 (2010)].

${ }^{55}$ A. Twardowski, P. Swiderski, M. von Ortenberg, and R. Pauthenet, Solid State Commun. 50, 509 (1984).

${ }^{56}$ S. A. Tarasenko, Phys. Rev. B 73, 115317 (2006). 